\title{
Chlorahololides A and B, Two Potent and Selective Blockers of Potassium Channel Isolated from Chloranthus holostegius
}

Sheng-Ping Yang, Zhao-Bing Gao, Fang-Dao Wang, Shang-Gao Liao, Hua-Dong Chen, Chuan-Rui Zhang, Guo-Yuan Hu and Jian-Min Yue*

State Key Laboratory of Drug Research, Shanghai Institute of Materia Medica, Shanghai Institutes for Biological Sciences, Chinese Academy of Sciences, 555 Zu Chong Zhi Road, Zhangjiang Hi-Tech Park, Shanghai, 201203, P. R. China

\section{Supporting Information}

S3. $\quad$ NMR data (Table 1) for chlorahololides A (1) and B (2).

S4. Experiment Section.

Figure S5. Key ROESY correlations $(\leftrightarrow)$ and important pyridine-induced solvent shifts $(\cdots)$ of chlorahololide B (2).

Figure S6. Concentration-inhibition curves of chlorahololides A-B (1-2).

Figure S7. $\quad{ }^{1} \mathrm{H}$ NMR (Varian Mercury-400, $400 \mathrm{MHz}, \mathrm{CDCl}_{3}$ ) of chlorahololide A (1).

Figure S8. ${ }^{13} \mathrm{C}$ NMR (Varian Mercury-400, $100 \mathrm{MHz}, \mathrm{CDCl}_{3}$ ) of chlorahololide A (1).

Figure S9. EI MS (Finnigan MAT 95) of chlorahololide A (1).

Figure S10. ESI MS (positive, Esquire3000plus) of chlorahololide A (1).

Figure S11. ESI MS (negative, Esquire3000plus) of chlorahololide A (1).

Figure S12. ${ }^{1} \mathrm{H}^{-1} \mathrm{H}$ COSY (Varian Mercury-400, $400 \mathrm{MHz}, \mathrm{CDCl}_{3}$ ) of chlorahololide A (1).

Figure S13. HSQC (Varian Mercury-400, $400 \mathrm{MHz}, \mathrm{CDCl}_{3}$ ) of chlorahololide A (1).

Figure S14. HMBC (Varian Mercury-400, $400 \mathrm{MHz}, \mathrm{CDCl}_{3}$ ) of chlorahololide A (1).

Figure S15. ROESY (Varian Inova-600, $600 \mathrm{MHz}, \mathrm{CDCl}_{3}$ ) of chlorahololide A (1).

Figure S16. IR (Perkin-Elmer 577, KBr disc) of chlorahololide A (1).

Figure S17. ${ }^{1} \mathrm{H}$ NMR (Varian Mercury-400, $400 \mathrm{MHz}, \mathrm{CDCl}_{3}$ ) of chlorahololide B (2).

Figure S18. ${ }^{1} \mathrm{H}$ NMR (Varian Mercury-400, $400 \mathrm{MHz}$, pyridine- $\left.d_{5}\right)$ ( $\left.\delta \quad 0.5-\delta \quad 7.1\right)$ of chlorahololide B (2).

${ }^{*}$ Corresponding author. Tel.: +86-21-50806718, Fax: +86-21-50806718, E-mail: jmyue@mail.shcnc.ac.cn 
Figure S19. ${ }^{1} \mathrm{H}$ NMR (Varian Mercury-400, $400 \mathrm{MHz}$, pyridine- $\left.d_{5}\right)$ ( $\delta$ 5.8- $\delta$ 12.2) of chlorahololide B (2).

Figure S20. ${ }^{13} \mathrm{C}$ NMR (Varian Mercury-400, $100 \mathrm{MHz}, \mathrm{CDCl}_{3}$ ) of chlorahololide B (2).

Figure S21. ESI MS (negative, Esquire3000plus) of chlorahololide B (2).

Figure S22. ESI MS (positive, Q-TOF Ultima) and HR ESI MS (positive, Q-TOF Ultima) of chlorahololide B (2).

Figure S23. ${ }^{1} \mathrm{H}-{ }^{1} \mathrm{H}$ COSY (Varian Mercury-400, $400 \mathrm{MHz}, \mathrm{CDCl}_{3}$ ) of chlorahololide B (2).

Figure S24. HSQC (Varian Mercury-400, $400 \mathrm{MHz}, \mathrm{CDCl}_{3}$ ) of chlorahololide B (2).

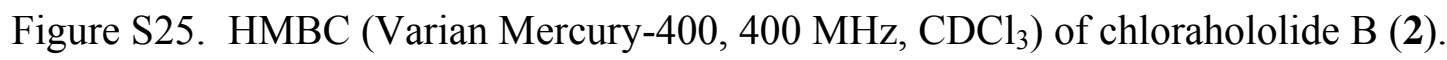

Figure S26. ROESY (Varian Inova-600, $600 \mathrm{MHz}, \mathrm{CDCl}_{3}$ ) of chlorahololide B (2).

Figure S27. IR (Perkin-Elmer 577, KBr disc) of chlorahololide B (2).

Figure S28. The atom serial numbers and molecular packing of chlorahololide A (1) in X-ray analysis.

S29. Crystal data and structure refinement (Table 2) for chlorahololide A (1) (label: ch2).

S30. Atomic coordinates and equivalent isotropic displacement parameters (Table 3) for chlorahololide A (1) (labeled as ch2).

S31-35. Bond lengths and angles (Table 4) for chlorahololide A (1) (labeled as ch2).

S36. Anisotropic displacement parameters (Table 5) for chlorahololide A (1) (labeled as $\operatorname{ch} 2)$.

S37. Hydrogen coordinatesand isotropic displacement parameters (Table 6) for chlorahololide A (1) (labeled as ch2).

S38-40. Torsion angles (Table 7) for chlorahololide A (1) (labeled as ch2).

S41. Hydrogen-bonds (Table 8) for chlorahololide A (1) (labeled as ch2).

S42-S44. Least-squares planesand deviations from them (Table 9) for chlorahololide A (1) (labeled as ch2). 
Table 1. NMR data for chlorahololides A (1) and B (2).

\begin{tabular}{|c|c|c|c|c|c|}
\hline & \multicolumn{2}{|r|}{1} & \multicolumn{3}{|c|}{2} \\
\hline & $\delta_{\mathrm{C}}{ }^{\mathrm{a}}$ & $\delta_{\mathrm{H}}{ }^{\mathrm{a}}$ & $\delta_{\mathrm{C}}{ }^{\mathrm{a}}$ & $\delta_{\mathrm{H}}{ }^{\mathrm{a}}$ & $\delta_{\mathrm{H}}^{\mathrm{b}}$ \\
\hline 1 & 28.6 & $1.56(\mathrm{~m})$ & 23.9 & 2.29 (ddd, 8.9, 6.7, 5.8) & $2.35(\mathrm{ddd}, 8.5,6.8,4.6)$ \\
\hline $2 \alpha$ & 9.0 & $0.92(\mathrm{ddd}, 8.5,8.4,5.9)$ & 8.9 & $1.01(\mathrm{ddd}, 8.9,8.5,6.3)$ & 0.97 (ddd, 8.6, 8.5, 5.7) \\
\hline $2 \beta$ & & $1.08(\mathrm{ddd}, 5.9,4.2,4.1)$ & & $1.19(\mathrm{ddd}, 6.3,5.8,4.3)$ & $1.44(\mathrm{~m})$ \\
\hline 3 & 30.0 & $1.88(\mathrm{ddd}, 10.2,6.2,4.1)$ & 29.8 & $1.80(\mathrm{~m})$ & $2.00(\mathrm{~m})$ \\
\hline 4 & 77.9 & & $77.0^{\mathrm{c}}$ & & \\
\hline 5 & 165.8 & & 160.4 & & \\
\hline 6 & 124.6 & & 123.4 & & \\
\hline 7 & 135.1 & & 147.8 & & \\
\hline 8 & 200.9 & & 94.0 & & \\
\hline $9 \alpha$ & 49.4 & $2.32(\mathrm{dd}, 17.1,0.7)$ & 200.5 & & \\
\hline $9 \beta$ & & $2.48(\mathrm{~d}, 17.1)$ & & & \\
\hline 10 & 44.7 & & 56.9 & & \\
\hline 11 & 135.2 & & 129.6 & & \\
\hline 12 & 170.4 & & 170.6 & & \\
\hline 13 & 19.3 & $1.57(\mathrm{~s}, 3 \mathrm{H})$ & 11.1 & $1.81(\mathrm{~s}, 3 \mathrm{H})$ & $1.93(\mathrm{~s}, 3 \mathrm{H})$ \\
\hline 14 & 21.9 & $1.25(\mathrm{~s}, 3 \mathrm{H})$ & 20.3 & $1.14(\mathrm{~s}, 3 \mathrm{H})$ & $1.10(\mathrm{~s}, 3 \mathrm{H})$ \\
\hline $15 \alpha$ & 41.4 & $1.77(\mathrm{~m})^{\mathrm{c}}$ & 40.1 & $1.74(\mathrm{dd}, 13.2,10.6)$ & $2.24(\mathrm{dd}, 13.2,10.9)$ \\
\hline $15 \beta$ & & $2.78(\mathrm{dd}, 16.2,7.0)$ & & $2.68(\mathrm{dd}, 13.2,6.7)$ & $3.04(\mathrm{dd}, 13.2,6.8)$ \\
\hline $1^{\prime}$ & 25.3 & $1.60(\mathrm{~m})$ & 26.4 & $1.55(\mathrm{~m})$ & $1.70(\mathrm{~m})$ \\
\hline $2^{\prime} \alpha$ & 16.3 & $0.81(\mathrm{ddd}, 9.7,9.5,5.2)$ & 9.8 & $0.64(\mathrm{ddd}, 9.2,8.5,5.2)$ & $0.65(\mathrm{ddd}, 8.9,8.8,4.8)$ \\
\hline $2^{\prime} \beta$ & & $0.66(\mathrm{ddd}, 5.2,3.6,3.4)$ & & $1.31(\mathrm{ddd}, 5.2,4.2,3.9)$ & $1.59(\mathrm{~m})$ \\
\hline $3^{\prime}$ & 23.9 & $1.96(\mathrm{~m})$ & 28.0 & $1.45(\mathrm{~m})$ & $1.55(\mathrm{~m})$ \\
\hline $4^{\prime}$ & 148.6 & & $77.0^{\mathrm{c}}$ & & \\
\hline $5^{\prime}$ & 58.3 & $2.53(\mathrm{ddd}, 10.9,2.8,2.7)$ & 55.3 & $2.15(\mathrm{dd}, 12.7,6.5)$ & $2.94(\mathrm{dd}, 12.3,7.3)$ \\
\hline $6^{\prime} \alpha$ & 63.8 & & 24.6 & $2.35(\mathrm{dd}, 18.9,6.5)$ & $3.41(\mathrm{dd}, 18.9,7.0)$ \\
\hline $6^{\prime} \beta$ & & $5.59(\mathrm{~d}, 10.9)$ & & $3.17(\mathrm{dd}, 18.9,12.7)$ & $3.65(\mathrm{dd}, 18.9,12.3)$ \\
\hline $7^{\prime}$ & 156.5 & & 174.3 & & \\
\hline $8^{\prime}$ & 86.6 & & 85.5 & & \\
\hline $9^{\prime}$ & 50.9 & $2.75(\mathrm{dd}, 14.1,7.0)$ & 51.4 & $2.58(\mathrm{dd}, 10.6,6.7)$ & $3.21(\mathrm{dd}, 10.9,6.8)$ \\
\hline $10^{\prime}$ & 41.7 & & 44.4 & & \\
\hline $11^{\prime}$ & 133.3 & & 123.6 & & \\
\hline $12^{\prime}$ & 172.6 & & 171.5 & & \\
\hline $13^{\prime} \mathrm{a}$ & 9.7 & $2.09(\mathrm{~s}, 3 \mathrm{H})$ & 53.5 & $5.42(\mathrm{~d}, 12.0)$ & $5.30(\mathrm{~d}, 12.3)$ \\
\hline $13^{\prime} \mathrm{b}$ & & & & $4.53(\mathrm{~d}, 12.0)$ & $4.92(\mathrm{~d}, 12.3)$ \\
\hline $14^{\prime}$ & 23.9 & $0.72(\mathrm{~s}, 3 \mathrm{H})$ & 23.8 & $0.96(\mathrm{~s}, 3 \mathrm{H})$ & $1.15(\mathrm{~s}, 3 \mathrm{H})$ \\
\hline $15^{\prime} \mathrm{a}$ & 108.6 & $5.01(\mathrm{dd}, 2.7,1.3)$ & 72.9 & $4.21(\mathrm{~d}, 11.2)$ & $4.99(\mathrm{~d}, 10.9)$ \\
\hline $15^{\prime} \mathrm{b}$ & & $4.67(\mathrm{dd}, 2.2,2.1)$ & & $4.06(\mathrm{~d}, 11.2)$ & $4.17(\mathrm{~d}, 10.9)$ \\
\hline $1^{\prime \prime}$ & & & 166.5 & & \\
\hline $2^{\prime \prime}$ & & & 111.9 & 5.48 (br d, 0.9) & $6.08(\mathrm{~d}, 1.4)$ \\
\hline $3^{\prime \prime}$ & & & 153.8 & & \\
\hline $4^{\prime \prime} \mathrm{a}$ & & & 67.4 & $4.77(\mathrm{dd}, 17.0,1.2)$ & $4.82(\mathrm{br} \mathrm{d}, 16.7)$ \\
\hline $4^{\prime \prime} b$ & & & & $4.27(\mathrm{~d}, 17.0)$ & $4.40(\mathrm{br} \mathrm{d}, 16.7)$ \\
\hline $5^{\prime \prime}$ & & & 15.1 & $2.10(\mathrm{~s}, 3 \mathrm{H})$ & $2.06(\mathrm{~s}, 3 \mathrm{H})$ \\
\hline $1^{\prime \prime \prime}$ & & & 172.0 & & \\
\hline $2^{\prime \prime \prime} \mathrm{a}$ & & & $28.3^{*}$ & $2.93(\mathrm{~m})^{*}$ & $2.81(\mathrm{~m})^{*}$ \\
\hline $2^{\prime \prime \prime} b$ & & & & $2.50(\mathrm{~m})^{*}$ & $2.71(\mathrm{~m})^{*}$ \\
\hline $3^{\prime \prime \prime} \mathrm{a}$ & & & $28.2^{*}$ & $2.75(\mathrm{~m})^{*}$ & $2.75(\mathrm{~m})^{*}$ \\
\hline $3^{\prime \prime \prime} \mathrm{b}$ & & & & $2.46(\mathrm{~m})^{*}$ & $2.65(\mathrm{~m})^{*}$ \\
\hline $4^{\prime \prime \prime}$ & & & 172.7 & & \\
\hline $\mathrm{OMe}$ & 52.3 & $3.70(\mathrm{~s}, 3 \mathrm{H})$ & & & \\
\hline $\mathrm{COMe}$ & 171.3 & & & & \\
\hline $\mathrm{COMe}$ & 20.6 & $2.13(\mathrm{~s}, 3 \mathrm{H})$ & & & \\
\hline $\mathrm{OH}$ & & 2.48 (br s) & & NB & 11.9 (br s) \\
\hline & & & & NB & 6.89 (br s) \\
\hline & & & & NB & 6.57 (br s) \\
\hline
\end{tabular}




\section{Experimental Section}

General experimental procedures. Melting points (uncorrected) were measured on a SGW® X-4 apparatus (Shanghai Precision \& Scientific Instrument Co., Ltd., China). Optical rotations were recorded on a Perkin-Elmer 341 polarimeter $(\mathrm{Na}$ filter, $\lambda=589 \mathrm{~nm})$. UV spectra were measured on a Shimadzu UV-2550 spectrophotometer. CD spectrum was measured on a JASCO J-810 instrument. IR spectra were recorded on a Perkin-Elmer 577 spectrometer with $\mathrm{KBr}$ disk. ${ }^{1} \mathrm{H}-,{ }^{13} \mathrm{C}$ NMR, ${ }^{1} \mathrm{H}-{ }^{1} \mathrm{H}$ COSY, HMBC, HSQC, HMQC and ROESY spectra were done on Varian Mercury-400 and Varian Inova-600 spectrometers and TMS as internal standard. EIMS (70 eV) and ESIMS were recorded on a Finnigan MAT 95 mass spectrometer, and an Esquire3000 plus and a Q-TOF Ultima (for HR ESIMS) instruments, respectively. All solvents used for chromatography were of analytical grade (Shanghai Chemical Plant). Silica gel (200-300 mesh), silica gel H, and Sephadex LH-20 (Pharmadex) were used for column chromatography, and percolated silica gel $\mathrm{GF}_{254}$ plates (Qingdao Haiyang Chemical Plant, Qingdao, People's Republic of China) were used for TLC. C18 reverse-phased silica gel (250 mesh, Merck) and MCI gel (CHP20P, 75-150 $\mu$, Mitsubishi Chemical Industries Ltd.) were also used for column chromatography.

Plant material. The plant material of Chloranthus holostegius (Hand.-Mazz.) Pei et Shan was collected from Xishuangbanna Tropical Botanical Garden (XTBG), Mengla County, Yunnan province, P. R. China in July 2005, and was authenticated by Prof. You-Kai Xu of Xishuangbanna Tropical Botanical Garden, Chinese Academy of Sciences. A voucher specimen has been deposited at Shanghai Institute of Materia Medica (Accession number: CH-2005-2Y).

Crystal structure measurement of chlorahololide A (1): Single-crystals $(0.40 \times 0.35 \times 0.35 \mathrm{~mm})$ used for X-ray analysis were crystallized in methanol. All measurements were done on a Rigaku AFC7R four circle diffractometer employing graphite monochromated Mo Ka radiation $(\lambda=$ $0.71073 \AA$ ) at $293 \mathrm{~K}$ and operating with the $\phi$ - $\omega$ scan mode. Crystal data: $\mathrm{C}_{33} \mathrm{H}_{36} \mathrm{O}_{8},\left(\mathrm{M}_{\mathrm{r}} 560.62\right)$, monoclinic, space group $\mathrm{C} 2, a=17.216(6) \AA, b=11.078(6) \AA, c=16.173(7) \AA, \alpha=90.00(4)^{\circ}, \beta=$ $108.63(4)^{\circ}, \gamma=90.00(4)^{\circ}, V=2923(2) \AA^{3}, Z=4, D_{\text {calcd }}=1.274 \mathrm{~g} / \mathrm{cm}^{3}, F(000)=1192$ and $\mu(\mathrm{Mo} \mathrm{Ka})$ $=0.091 \mathrm{~mm}^{-1}$. The structure was solved by direct methods (SHELXS-97) and refined with full-matrix least-squares calculations on $F^{2}$ using SHELXL-97. 


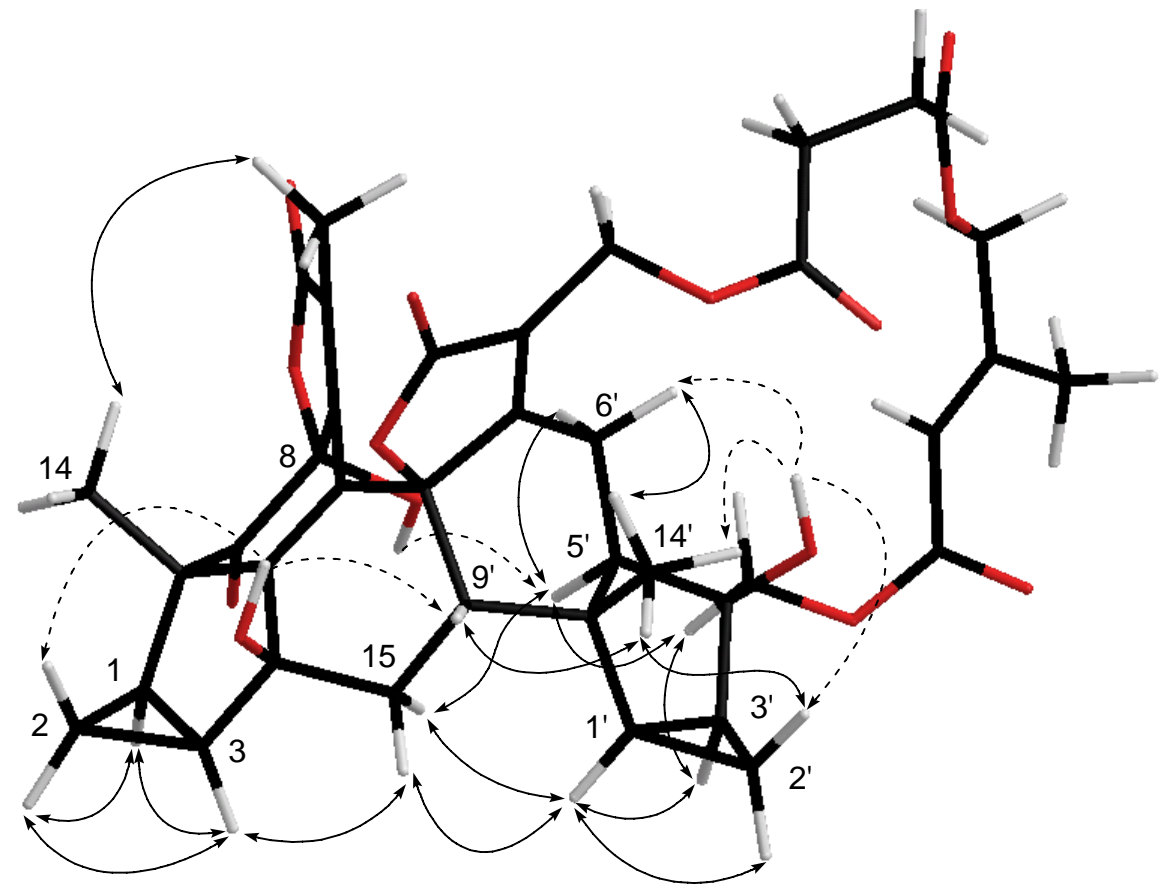

Key ROESY correlations $(\leftrightarrow)$ and important pyridine-induced solvent shifts $(\cdots)$ of chlorahololide B (2). 

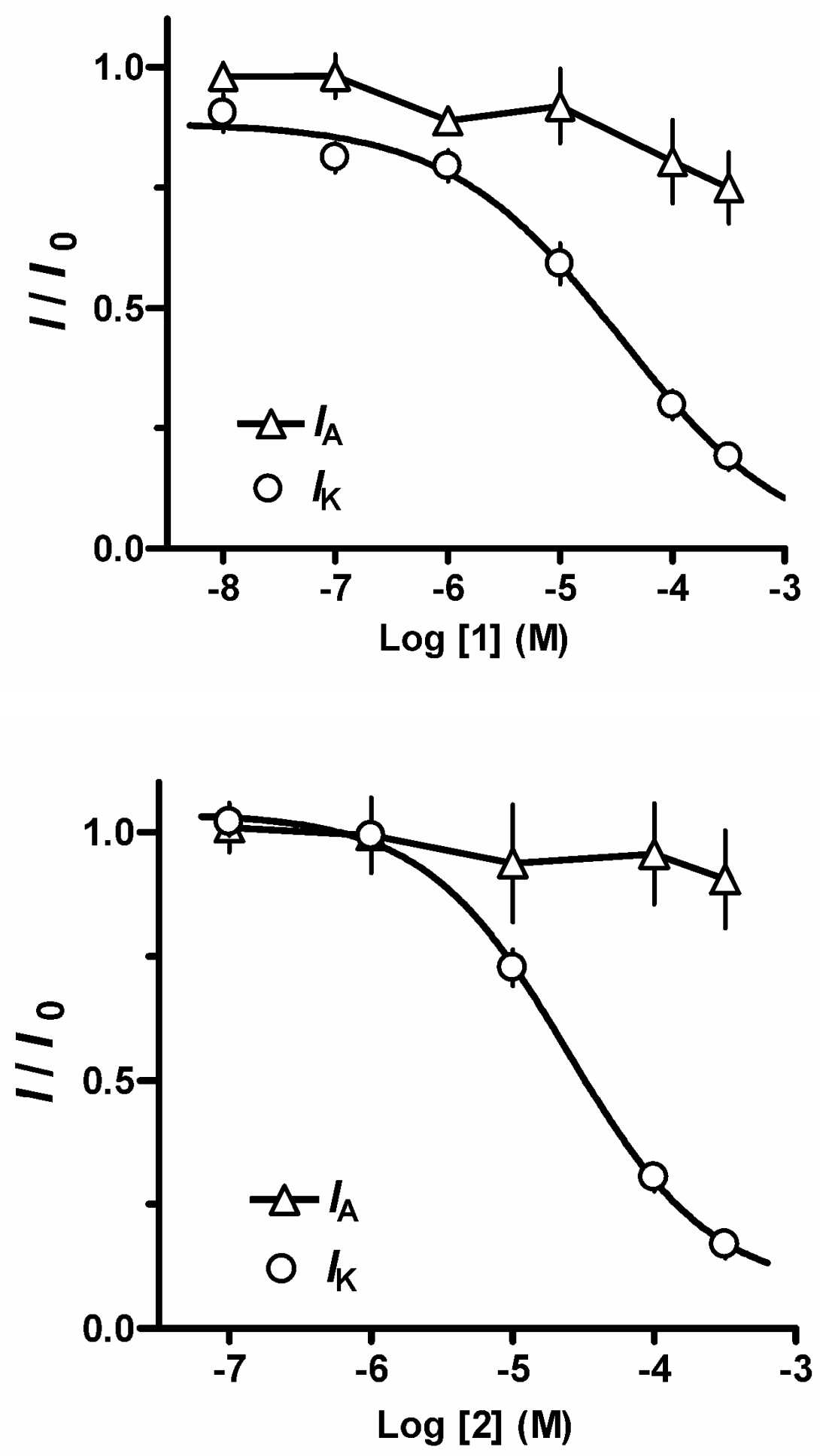

Concentration-inhibition curves of chlorahololides A-B (1-2) on voltage-gated potassium $\left(\mathrm{K}^{+}\right)$currents in rat hippocampal neurons. $I_{\mathrm{K}}$ and $I_{\mathrm{A}}$ : the delayed rectifier and fast transient $\mathrm{K}^{+}$currents, respectively. Each symbol represents the mean \pm SEM from 6 neurons. 
Figure S7. ${ }^{1} \mathrm{H}$ NMR of chlorahololide A (1)

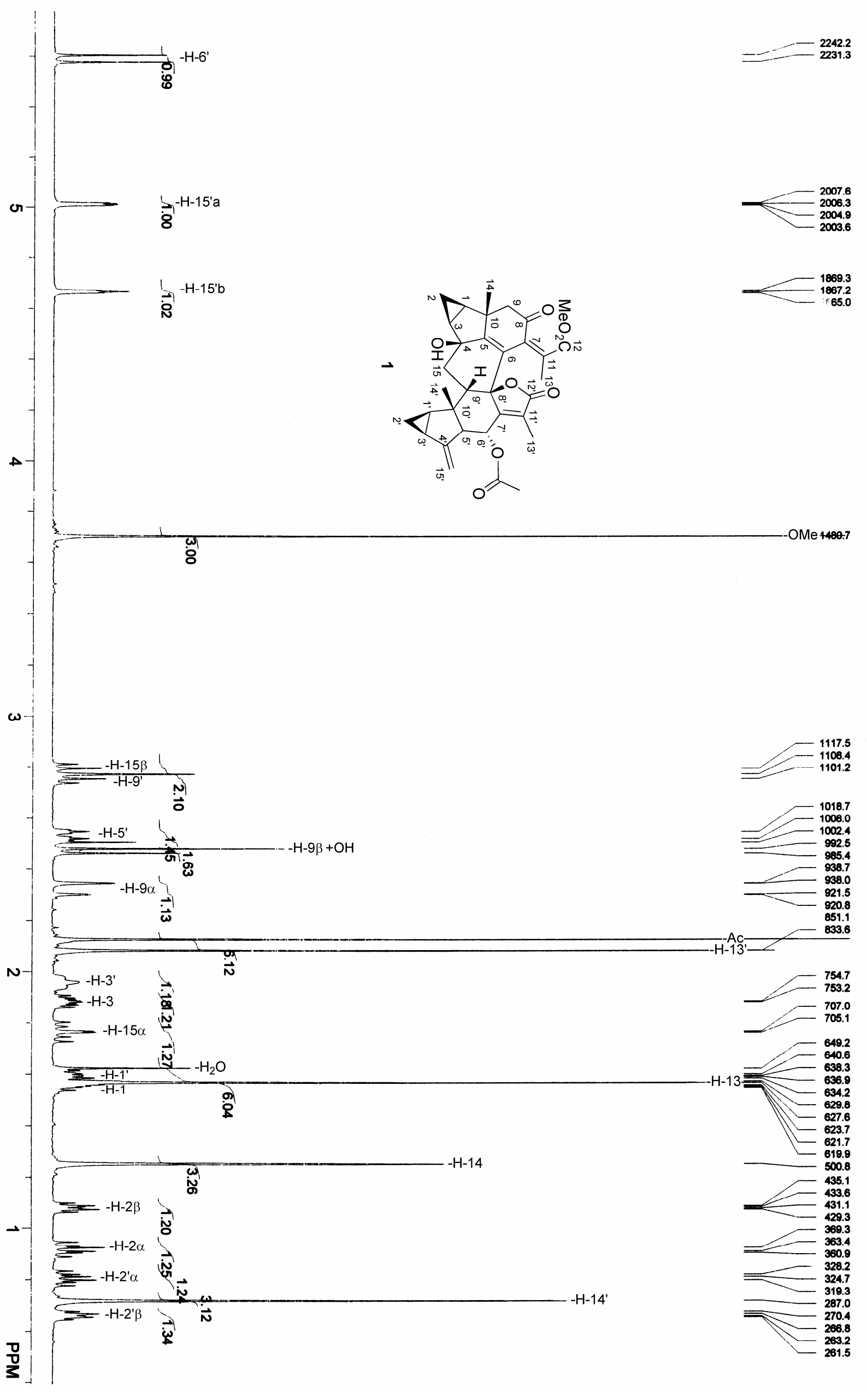


Figure S8. ${ }^{13} \mathrm{C}$ NMR of chlorahololide A (1)
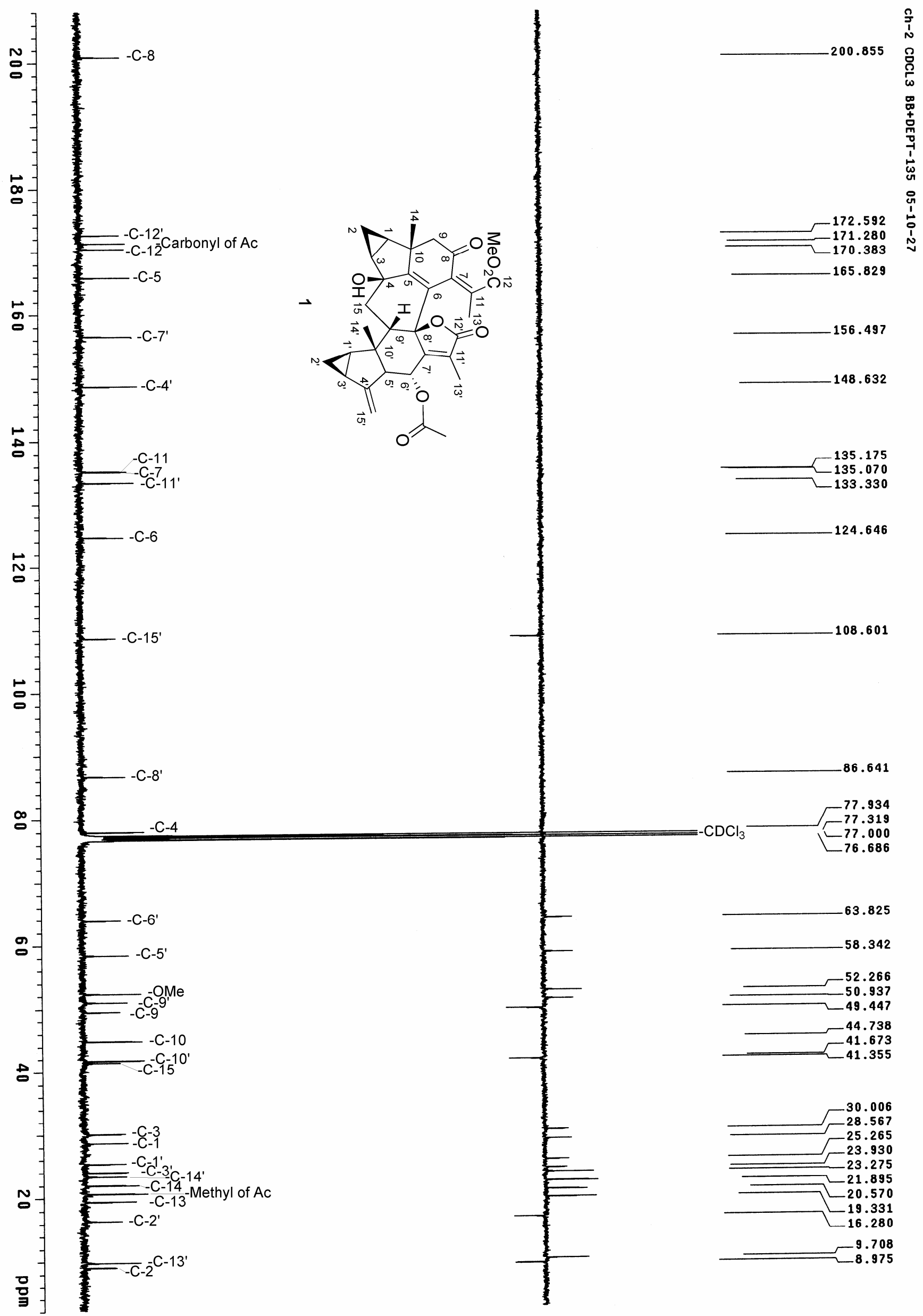


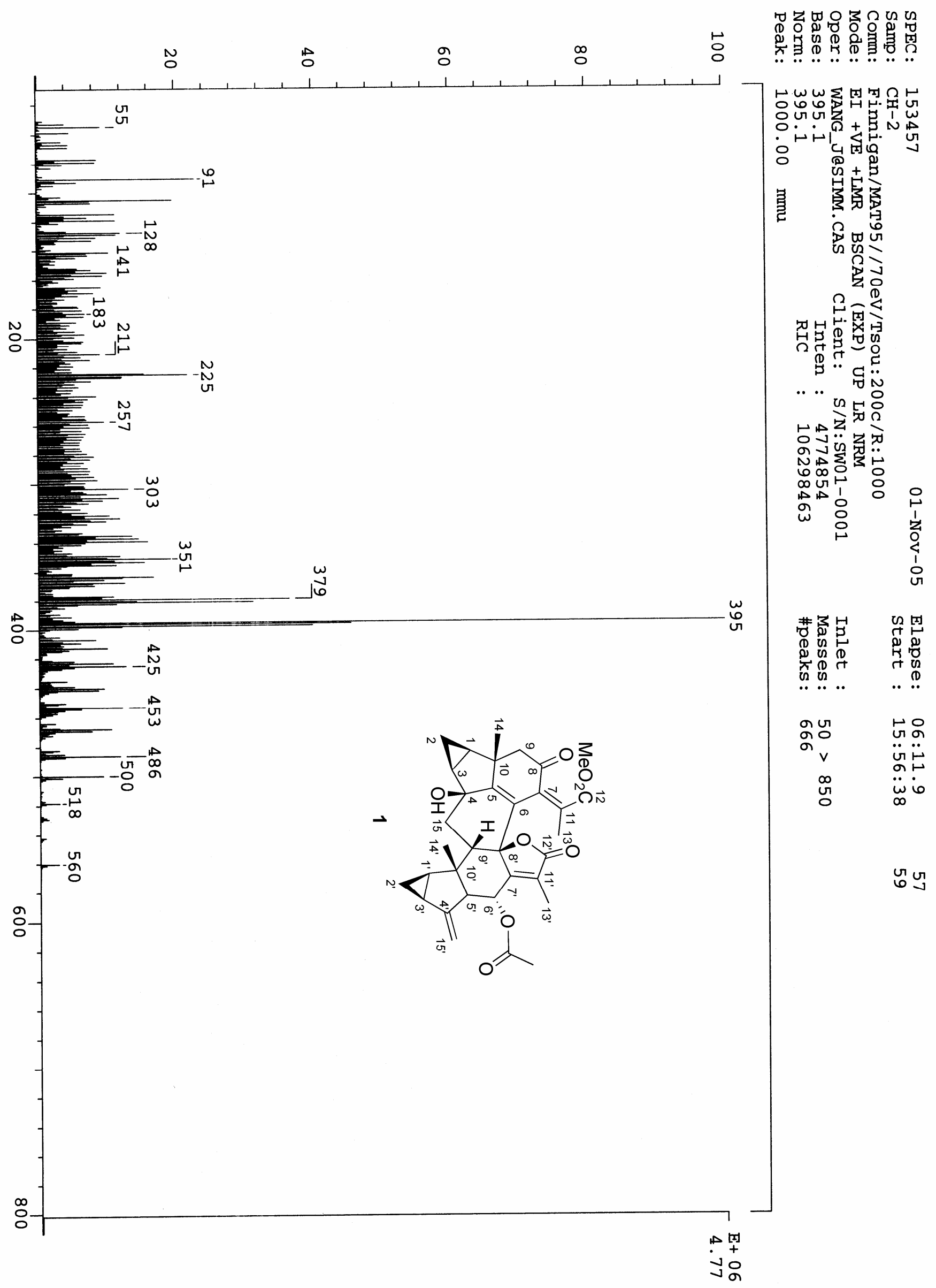




\section{Display Report}

\section{Analysis Info}

\section{Analysis Name}

Method

Sample Name

Comment
011-1601.D

Copy of SOPMSMSP.M

yjm- $\mathrm{CH}-2$

$>$
Acquisition Date 12/14/05 14:53:18

Operator Administrator

Instrument esquire3000plus_01005
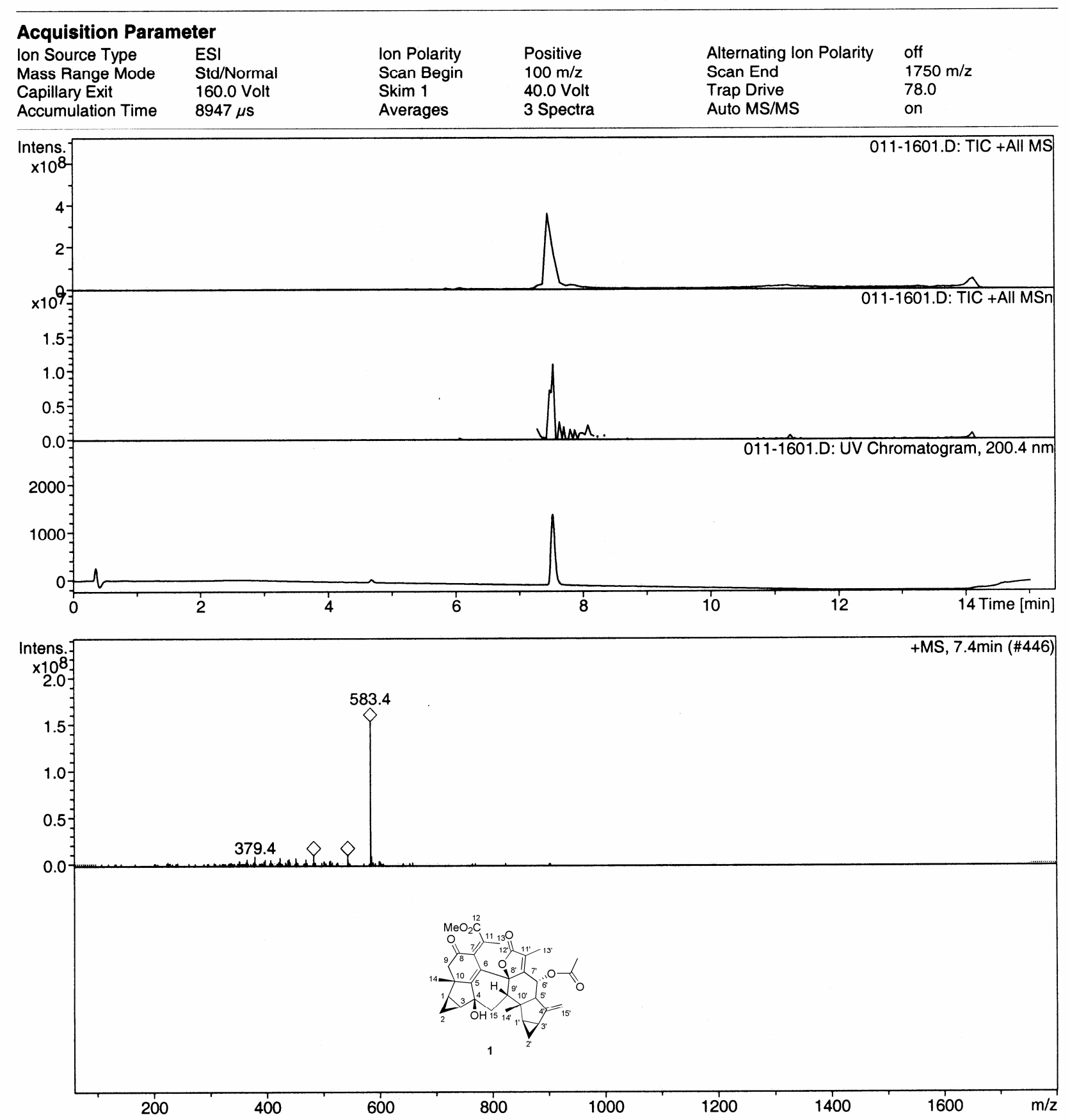


\section{Display Report}

\section{Analysis Info}

Analysis Name

Method

Sample Name

Comment
011-5901.D

Copy of SOPMSMSN.M

yjm- $\mathrm{CH}-2$

$>$
Acquisition Date

Operator

Instrument
12/15/05 03:08:54

Administrator

esquire3000plus_01005
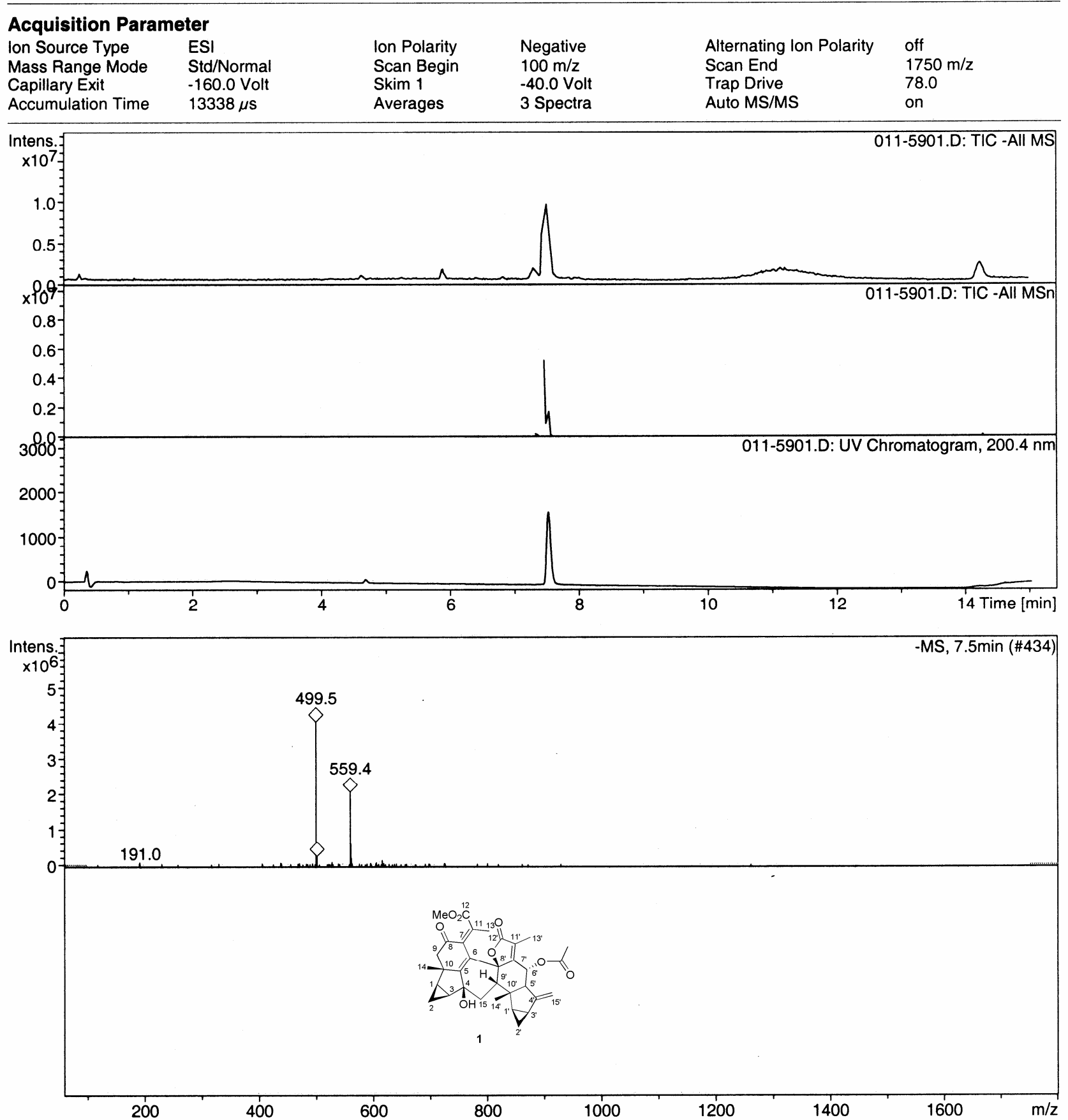
Figure S12. ${ }^{1} \mathrm{H}-{ }^{1} \mathrm{H}$ COSY of chlorahololide A (1)

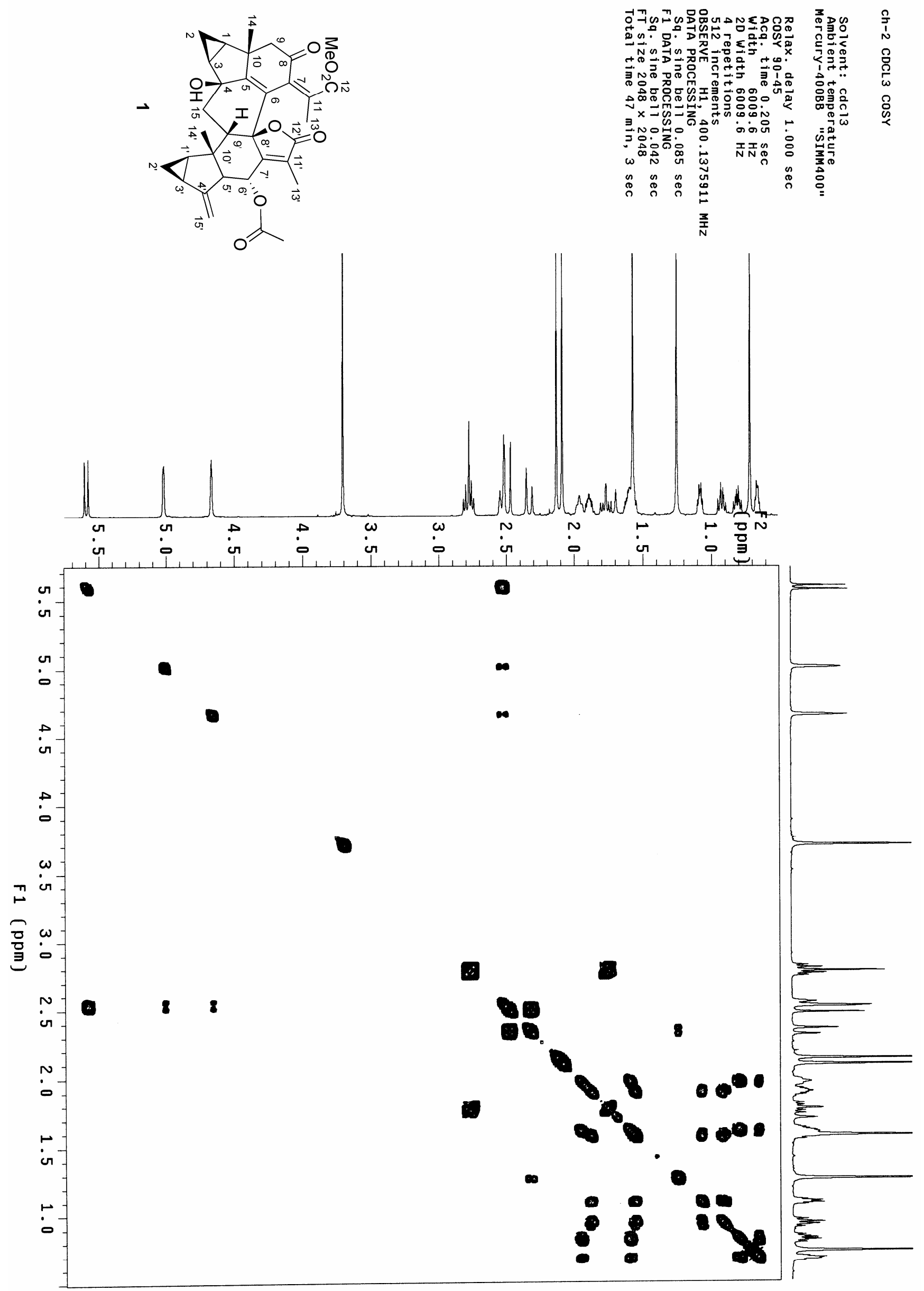


Figure S13. HSQC of chlorahololide A (1)

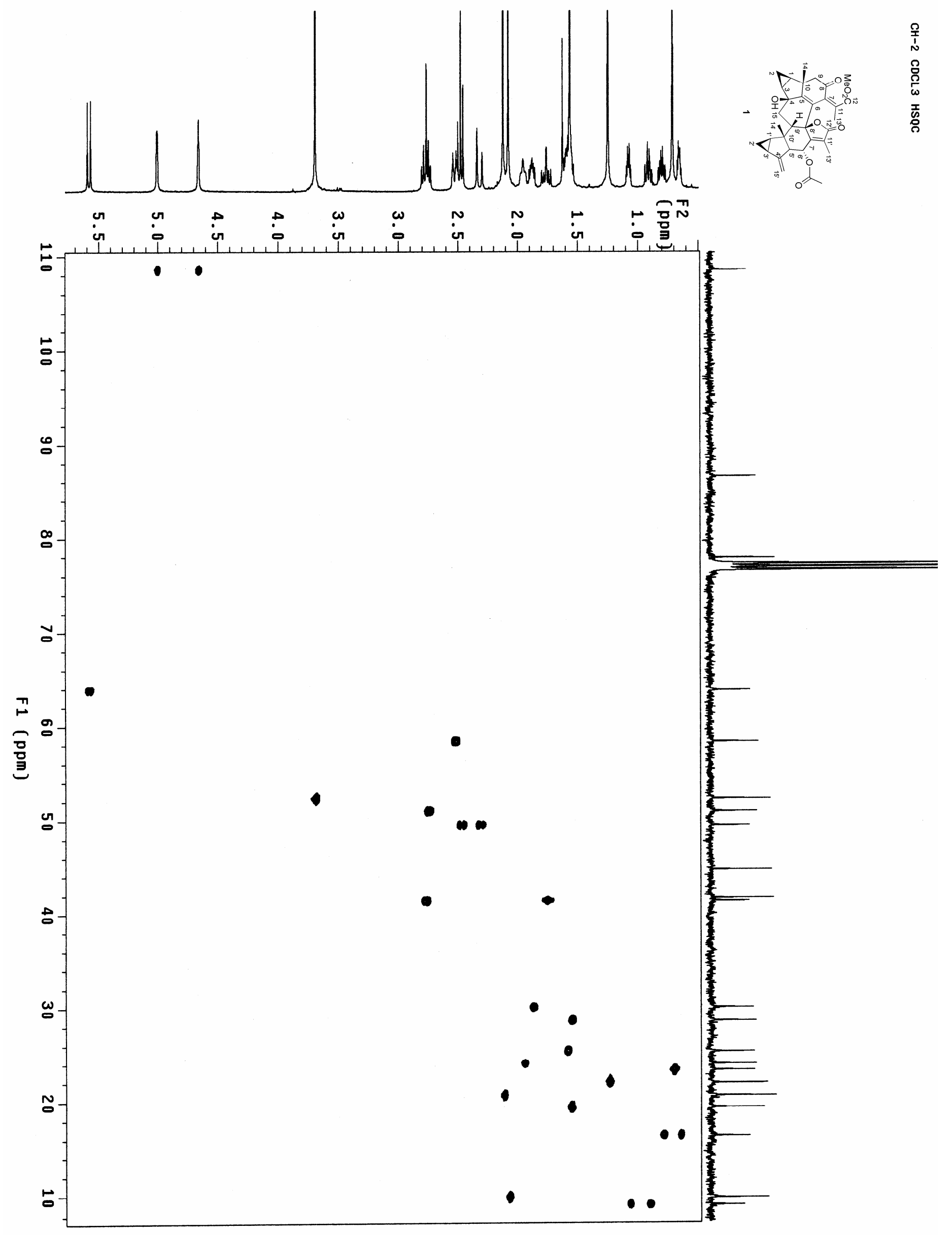


Figure S14. HMBC of chlorahololide A (1)

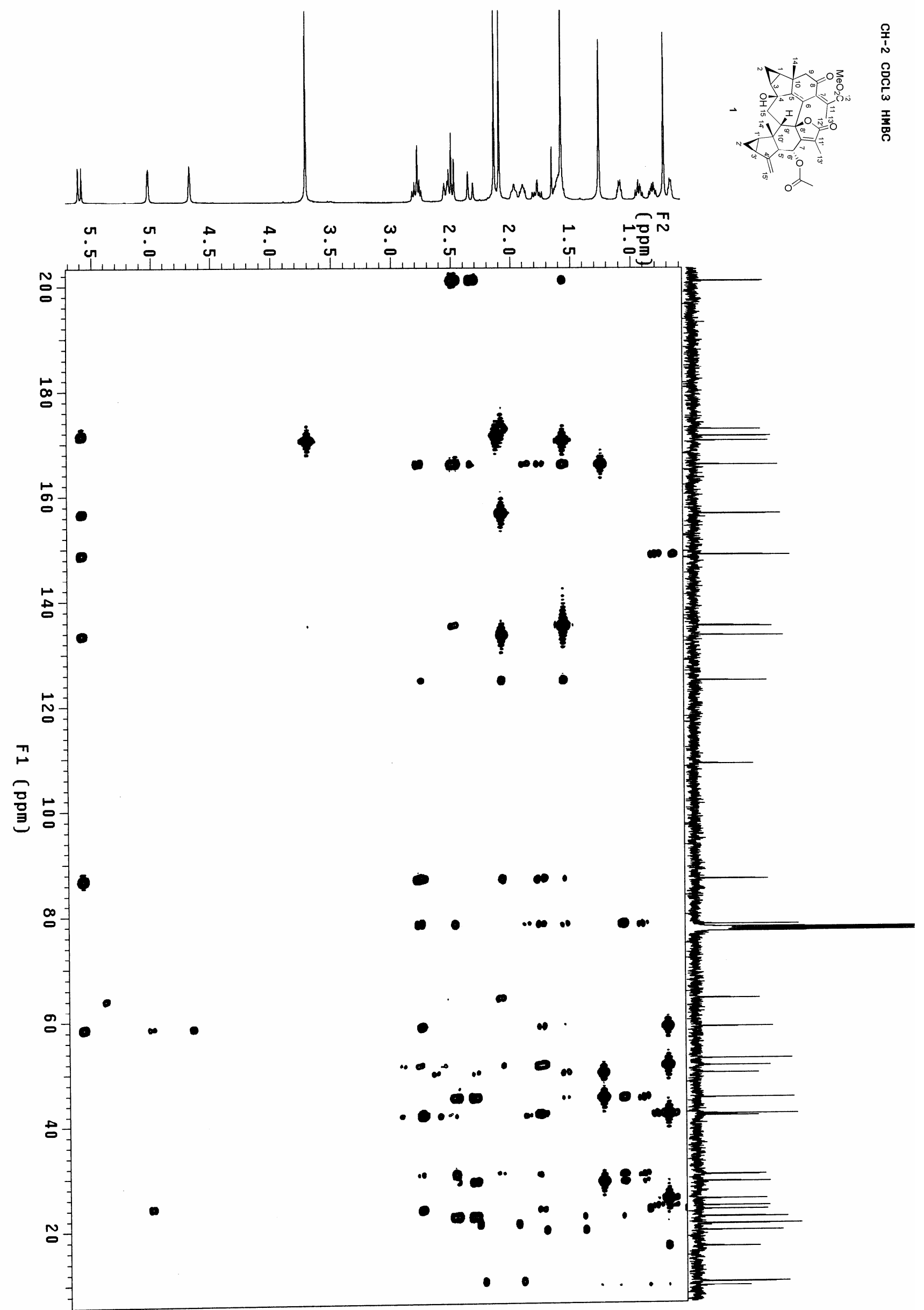


Figure S15. ROESY of chlorahololide A (1)

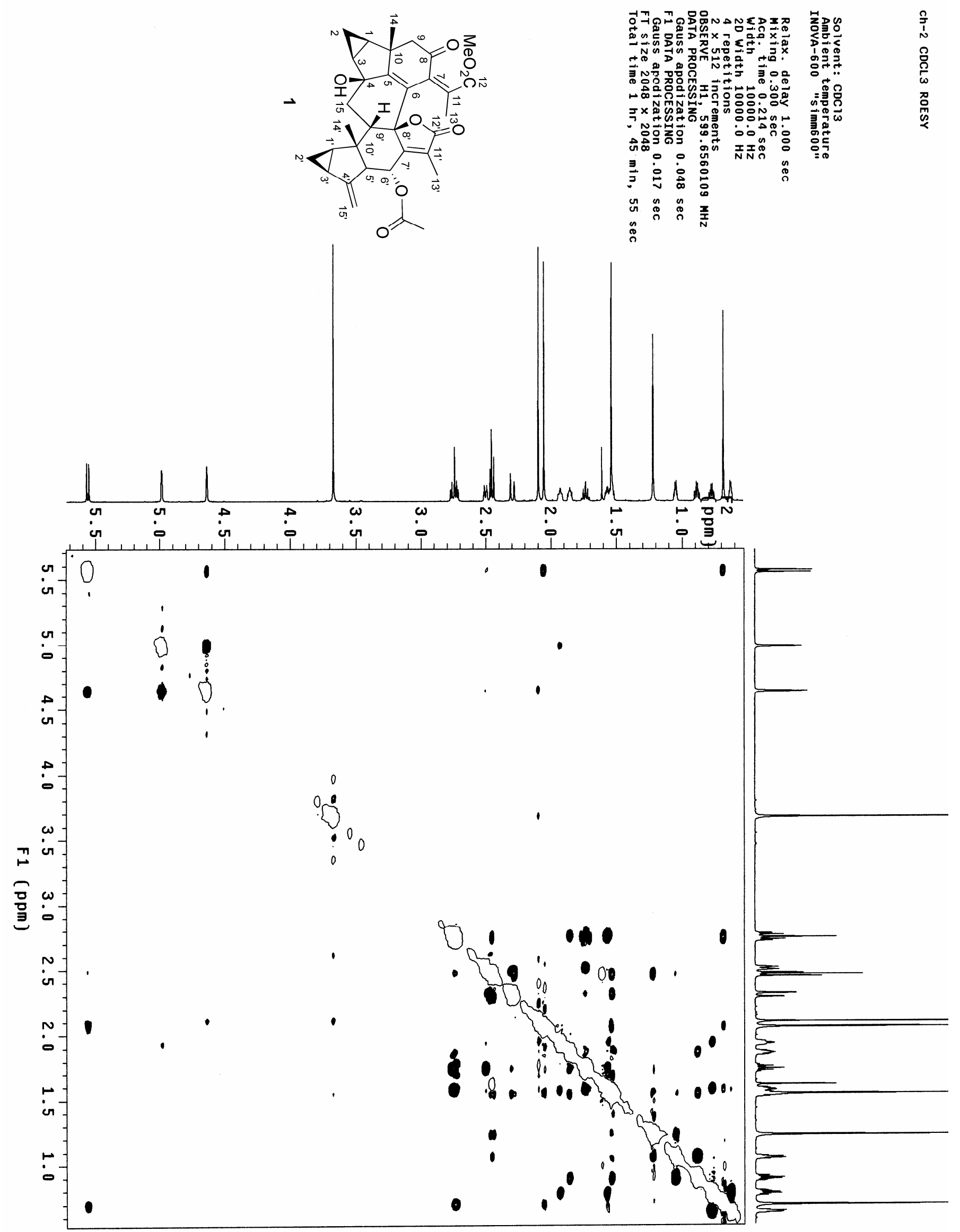




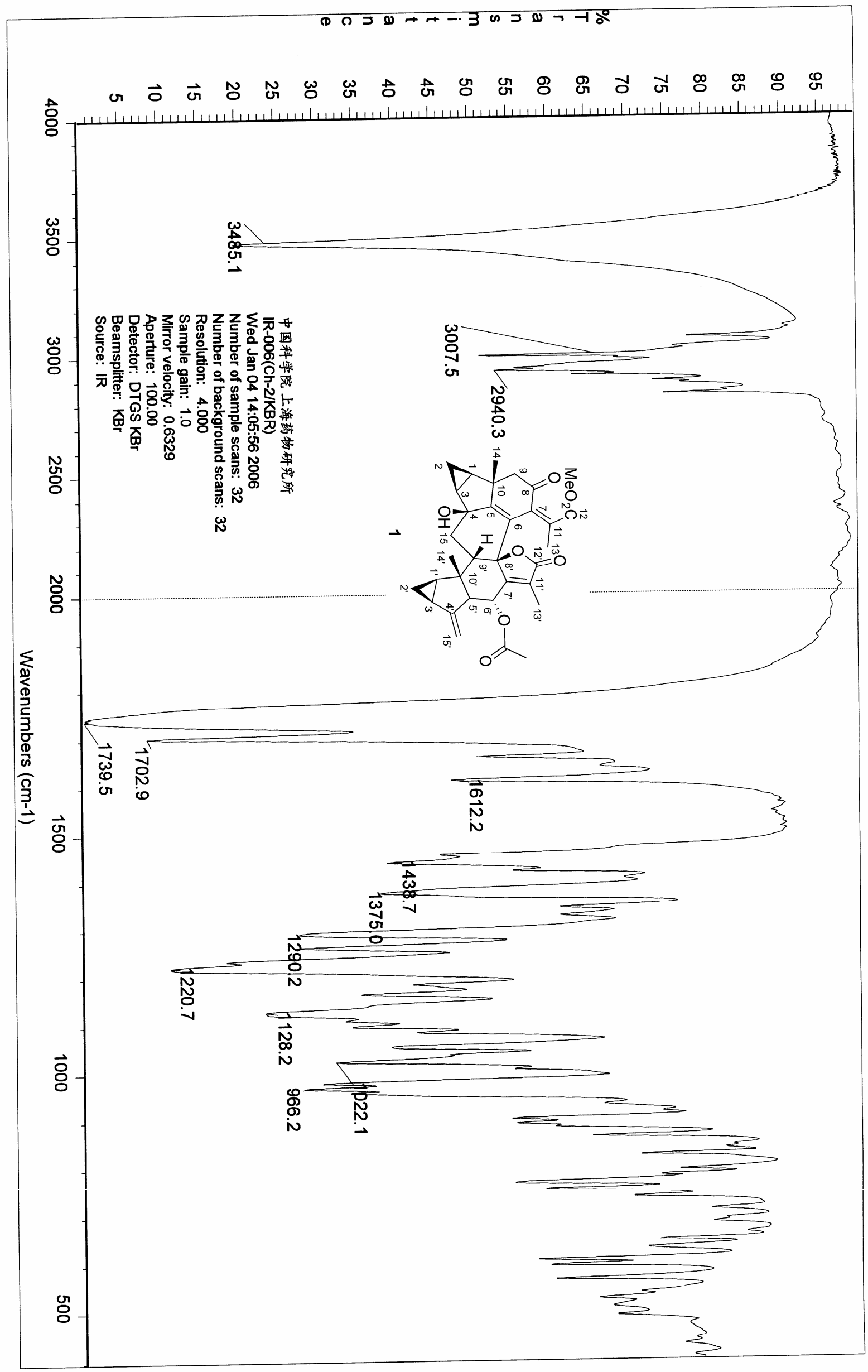


Figure S17. ${ }^{1} \mathrm{H}$ NMR of chlorahololide B (2)

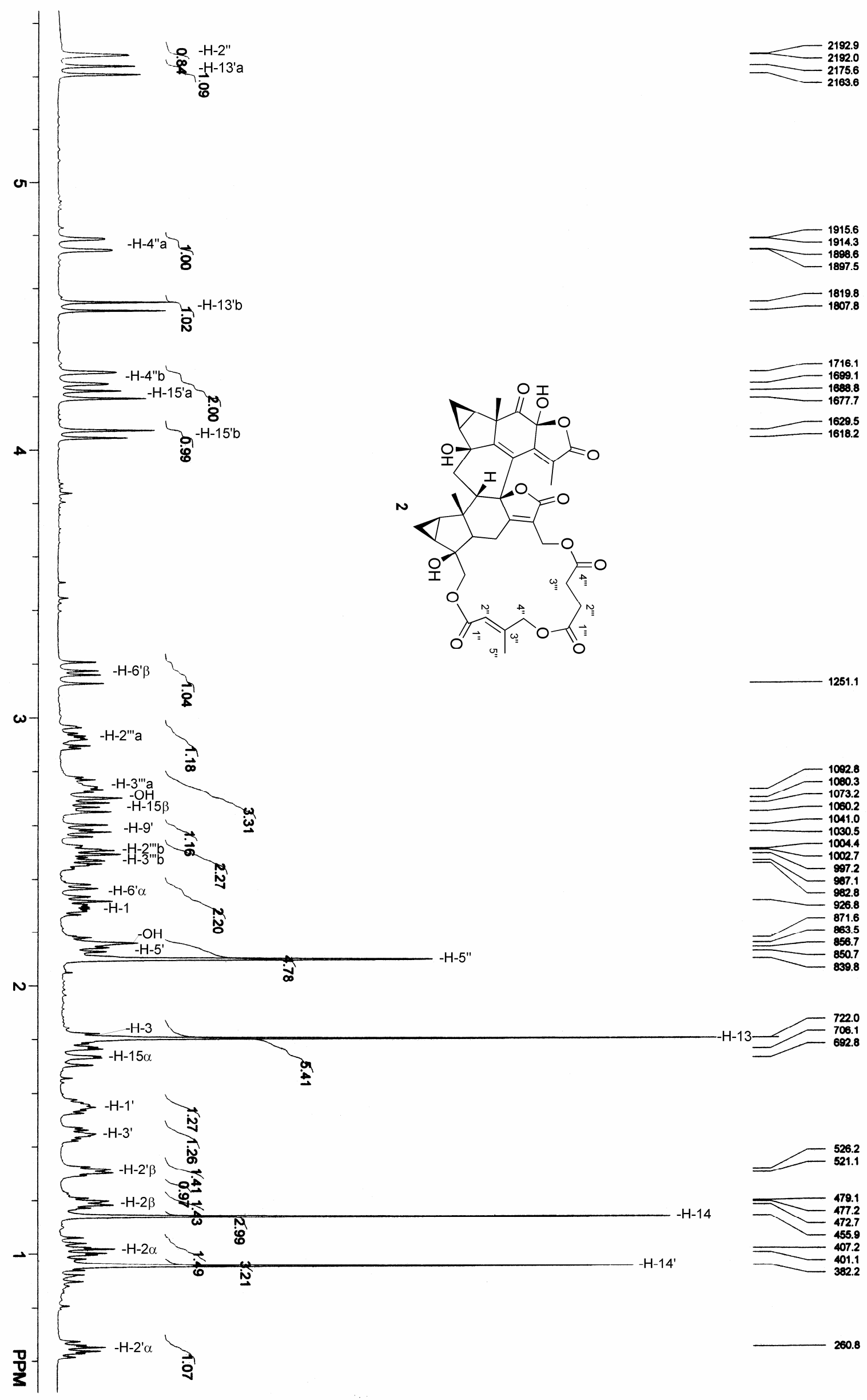


Figure S18. ${ }^{1} \mathrm{H}$ NMR (pyridine- $\left.d_{5}\right)$ of chlorahololide B (2) $(\delta 0.5-\delta 7.1)$

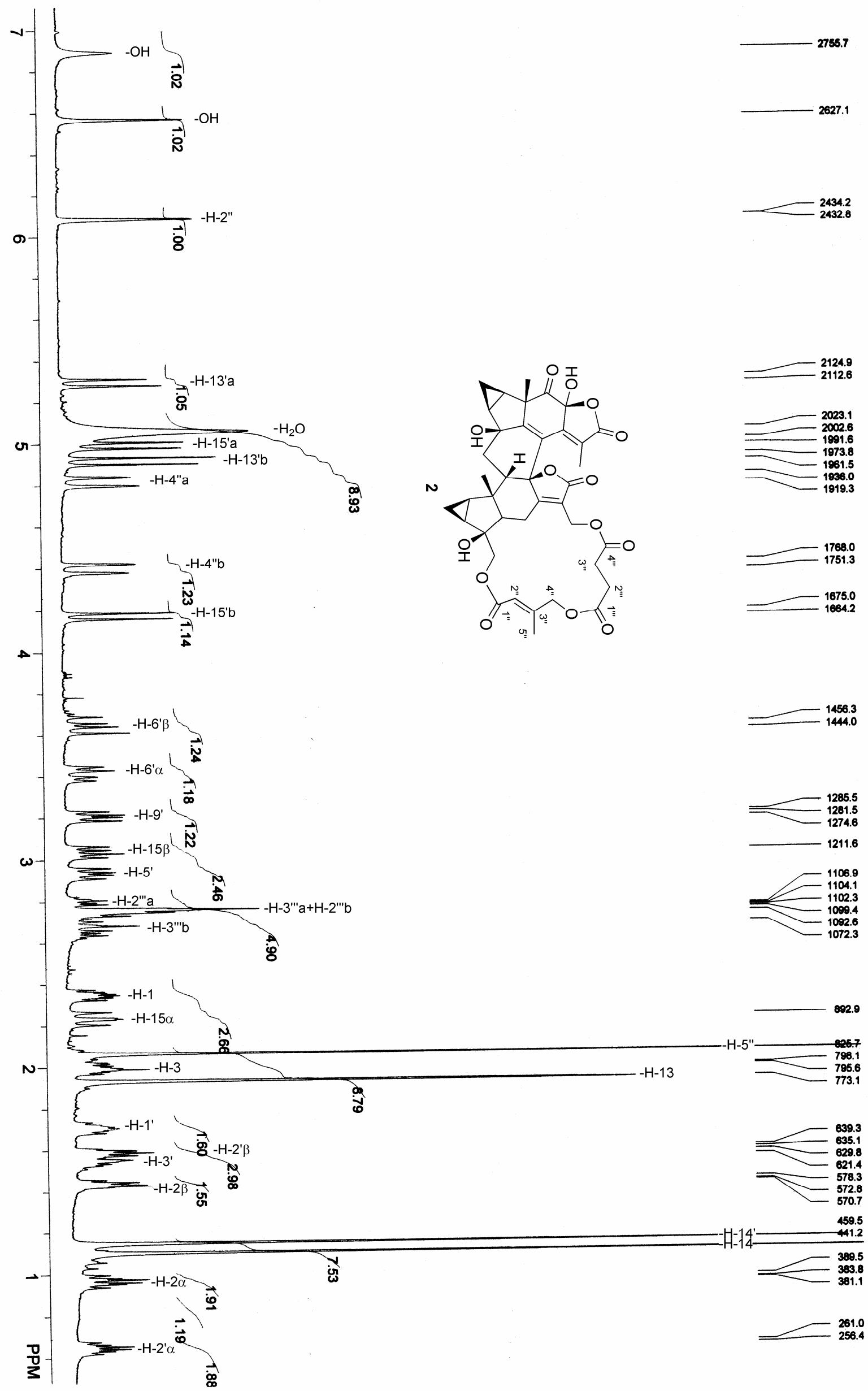


Figure S19. ${ }^{1} \mathrm{H}$ NMR (pyridine- $d_{5}$ ) of chlorahololide B (2) ( $\left.\delta 5.8-\delta 12.2\right)$

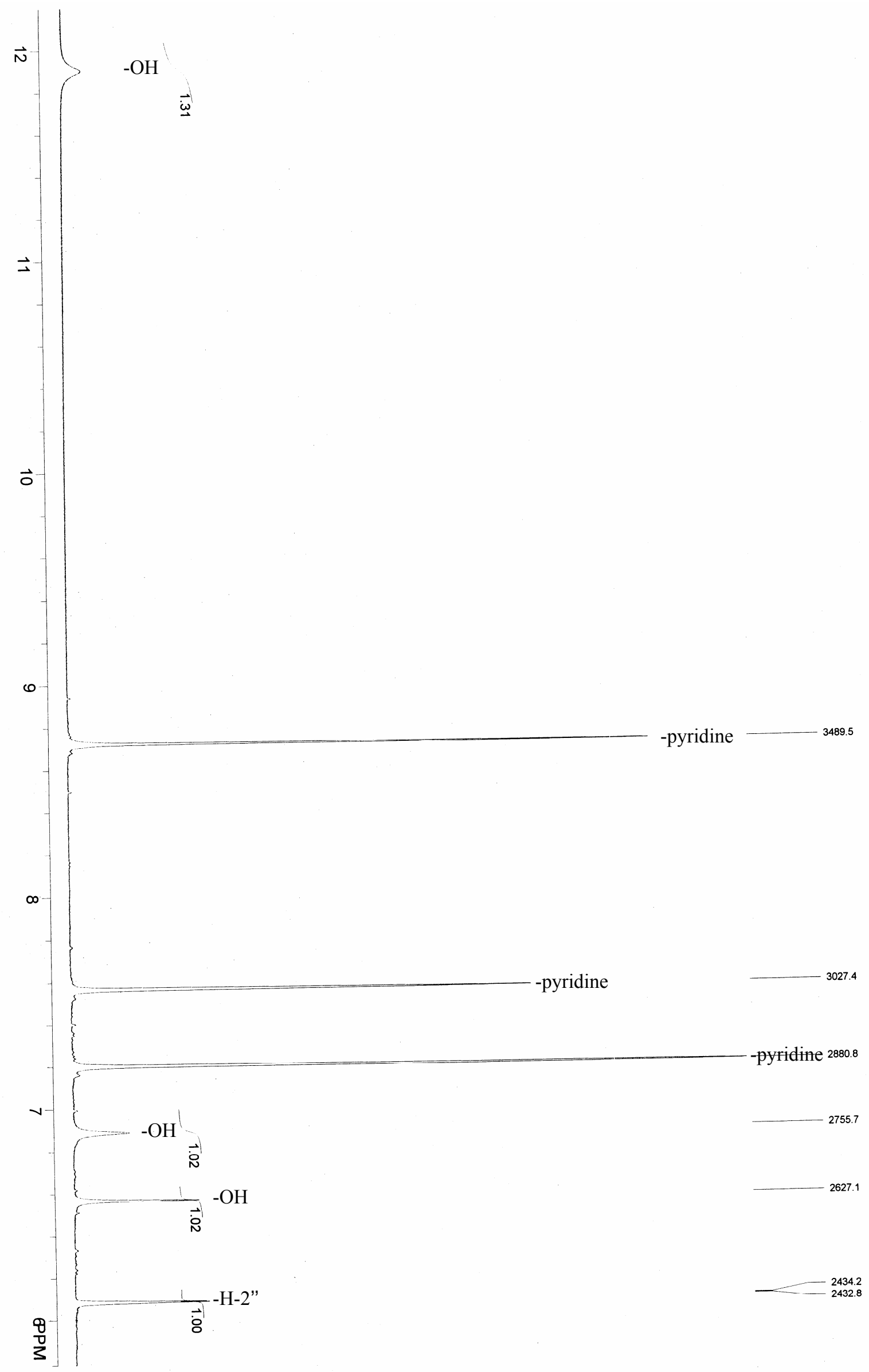


Figure S20. ${ }^{13} \mathrm{C}$ NMR of chlorahololide B (2)

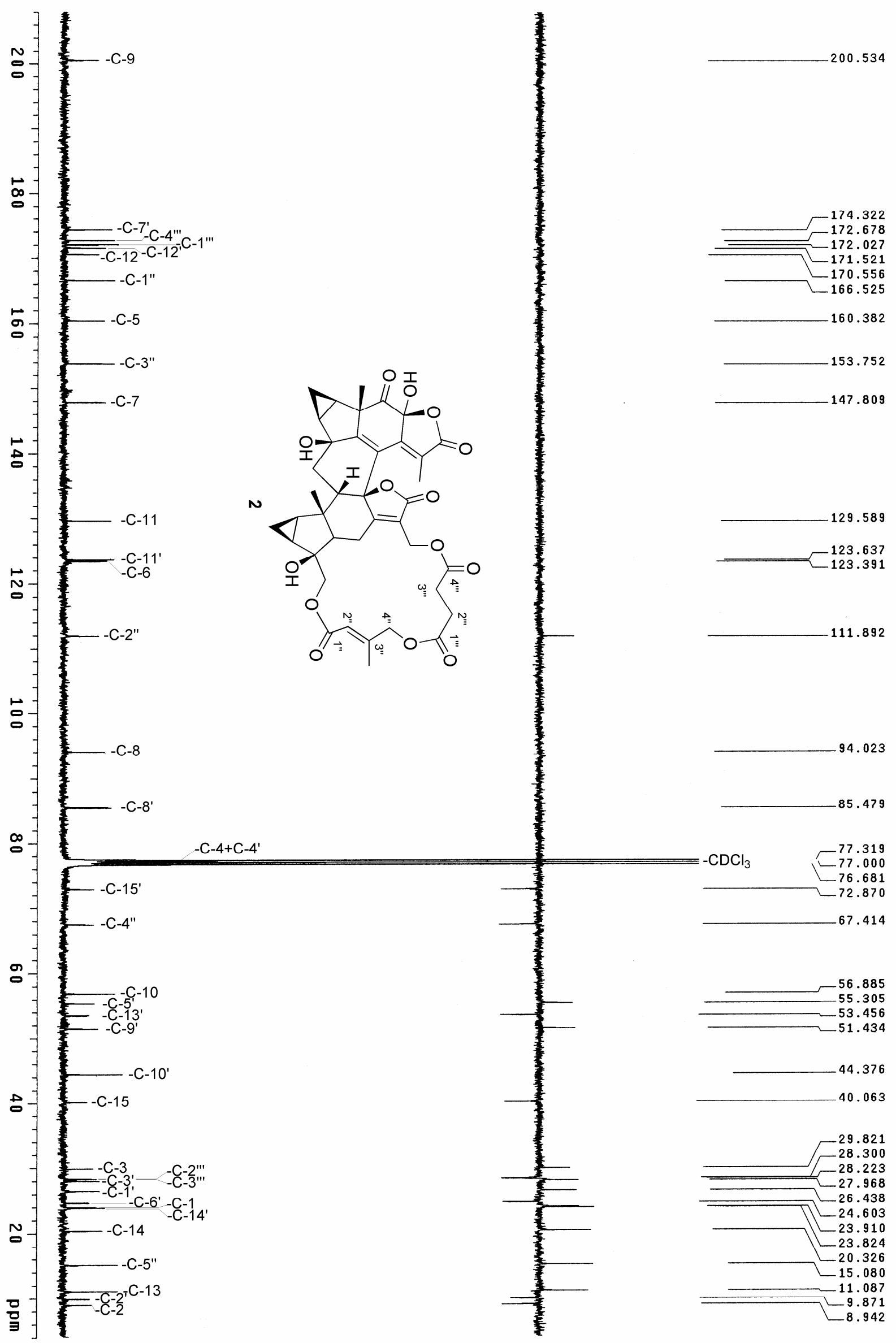




\section{Display Report}

\section{Analysis Info}

Analysis Name Method

Sample Name

Comment
063-br01.D

Copy of SOPMSMSN.M

ch-14

$>$
Acquisition Date 12/16/05 08:50:58

Operator Administrator

Instrument esquire3000plus_01005
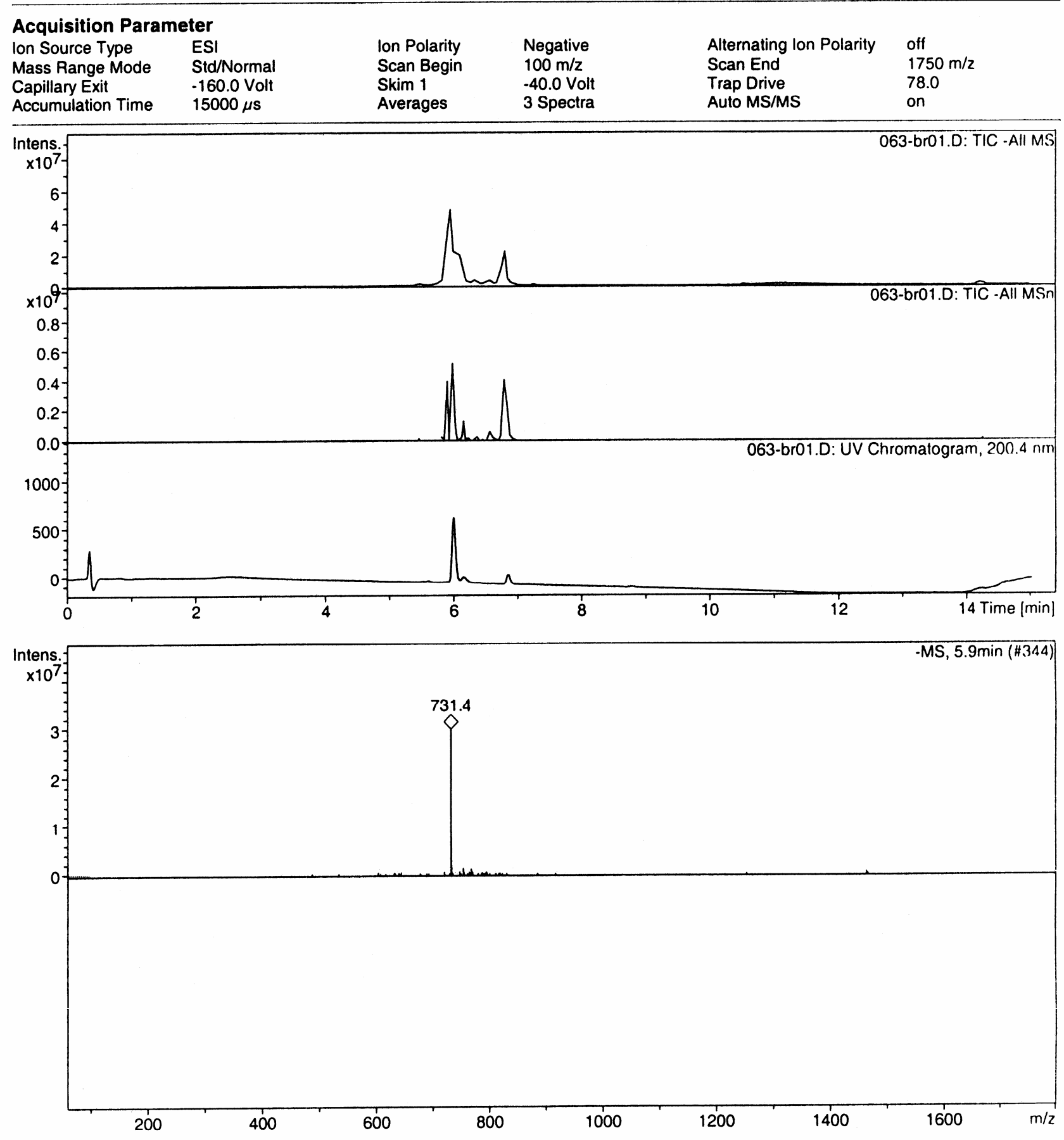
Figure S22. ESI MS (positive) and HR ESI MS (positive) of chlorahololide B (2)

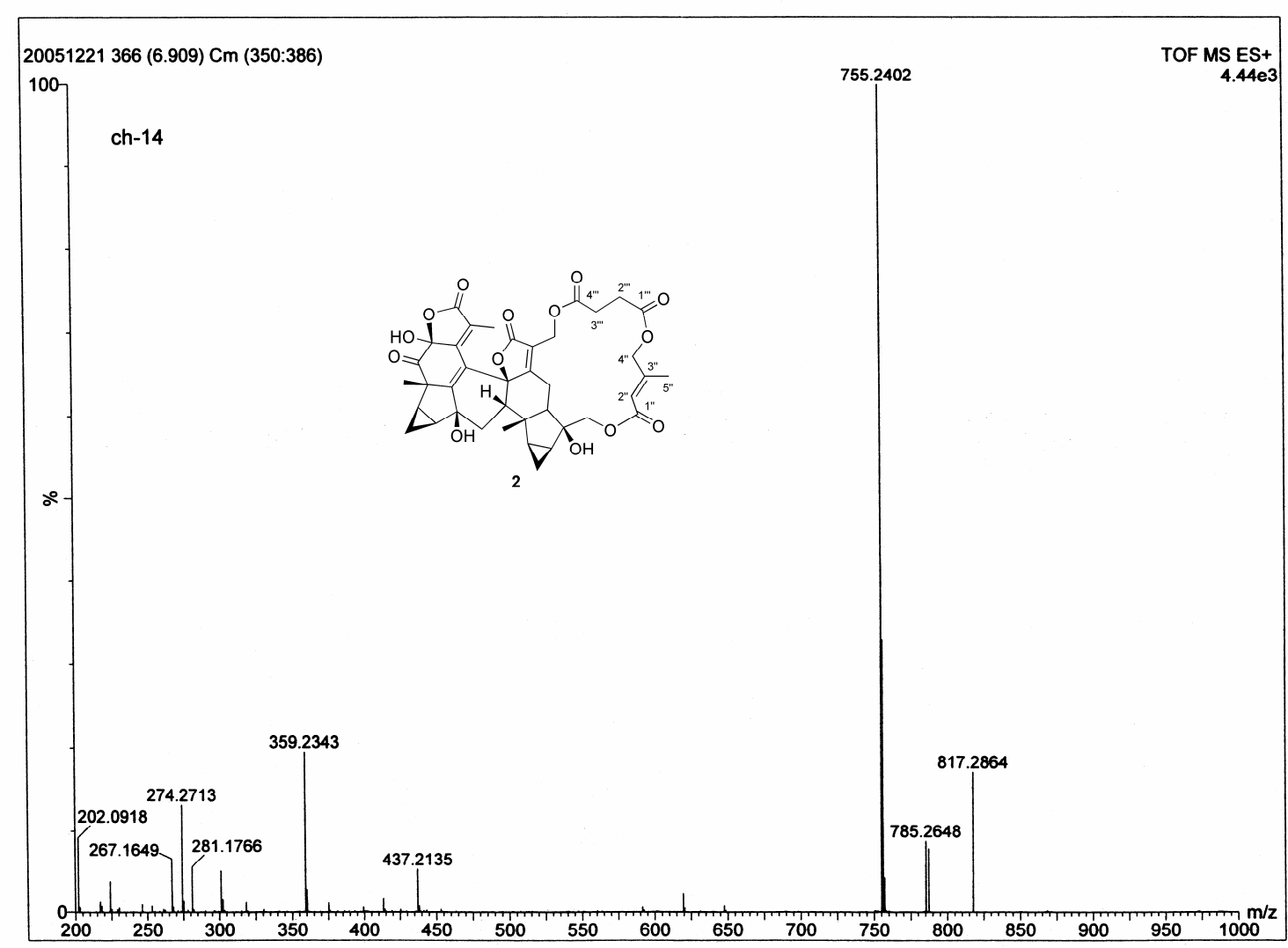

ESI MS (positive) of chlorahololide B (2)

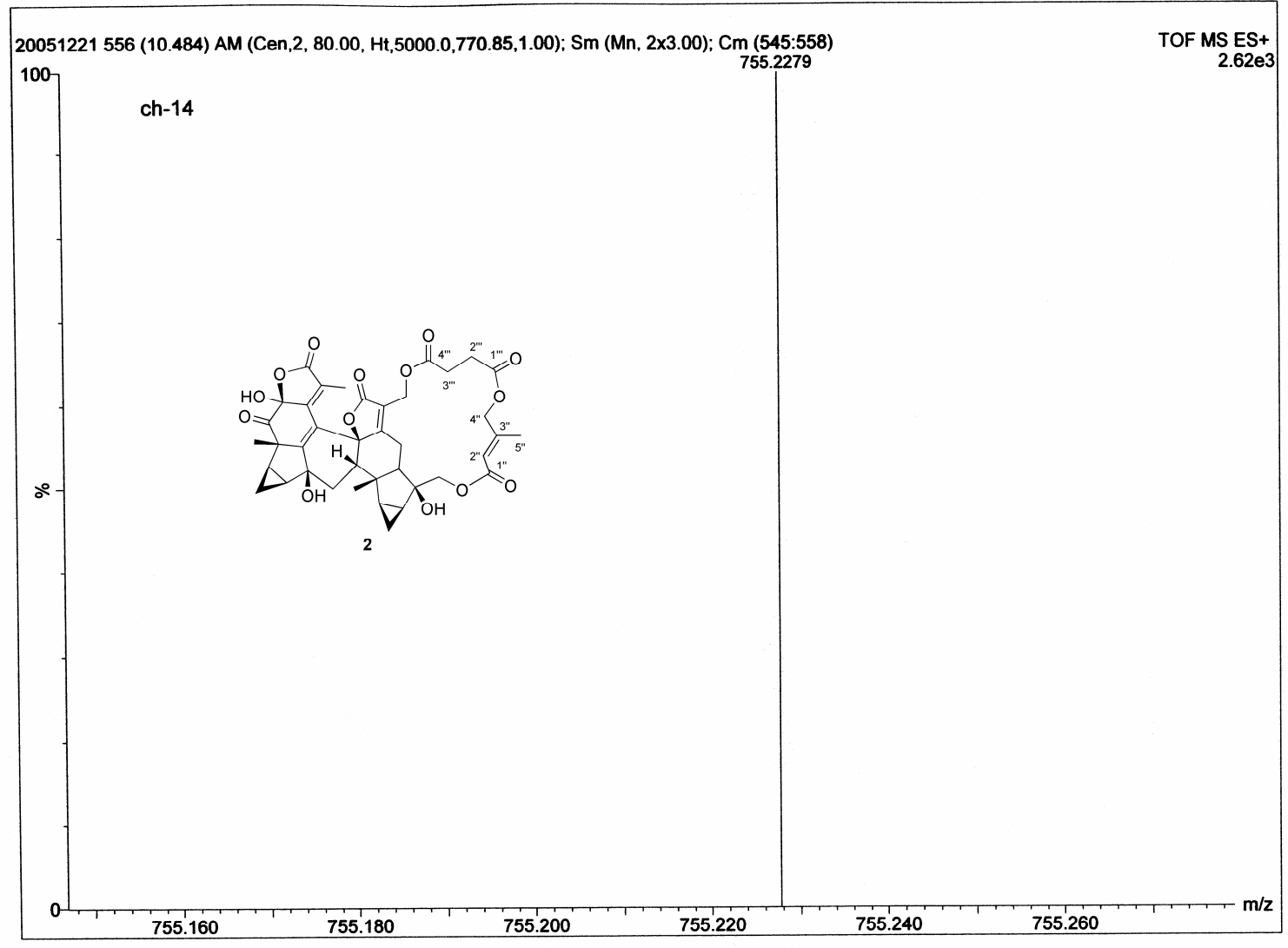

HR ESI MS (positive) of chlorahololide B (2) 


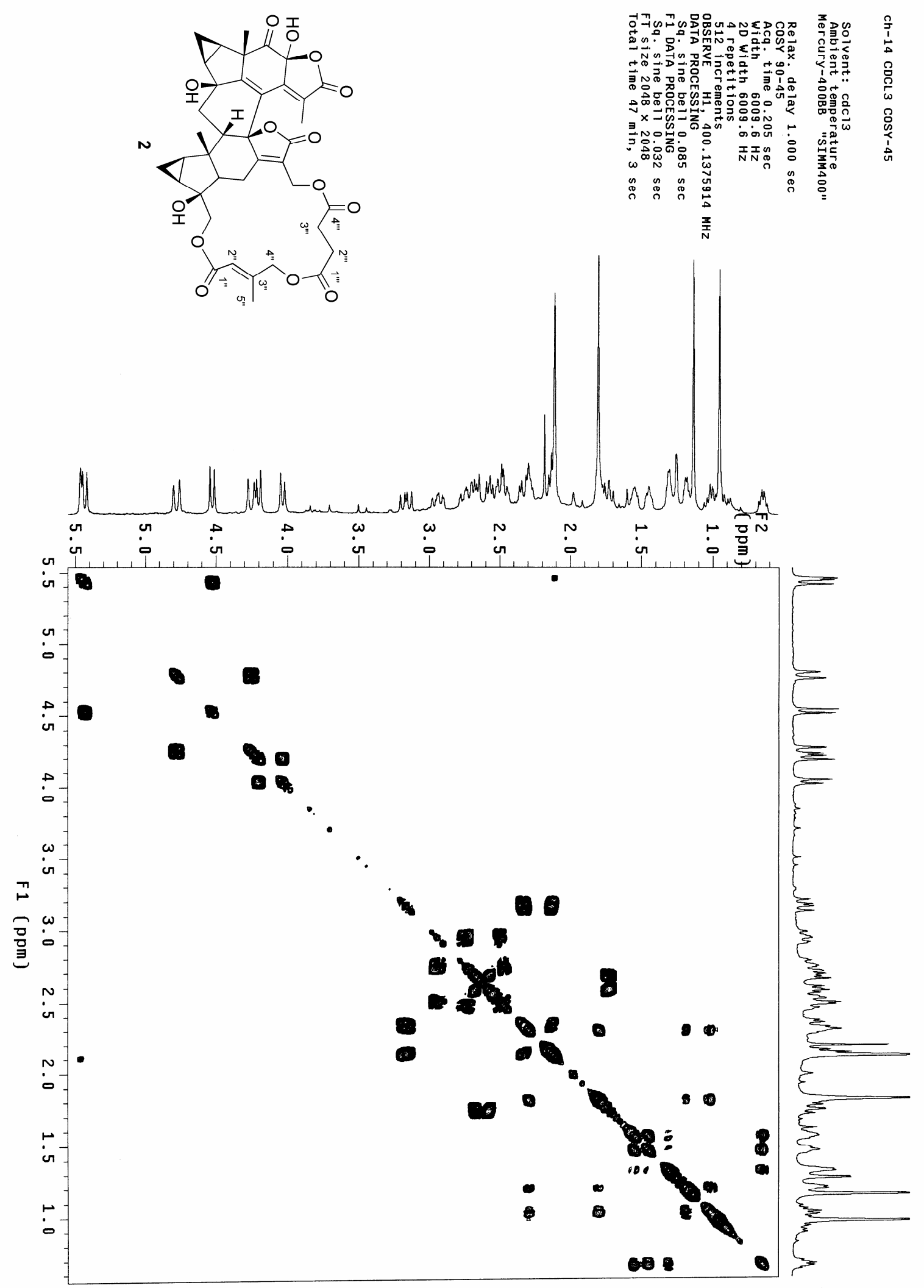


Figure S24. HSQC of chlorahololide B (2)

S24

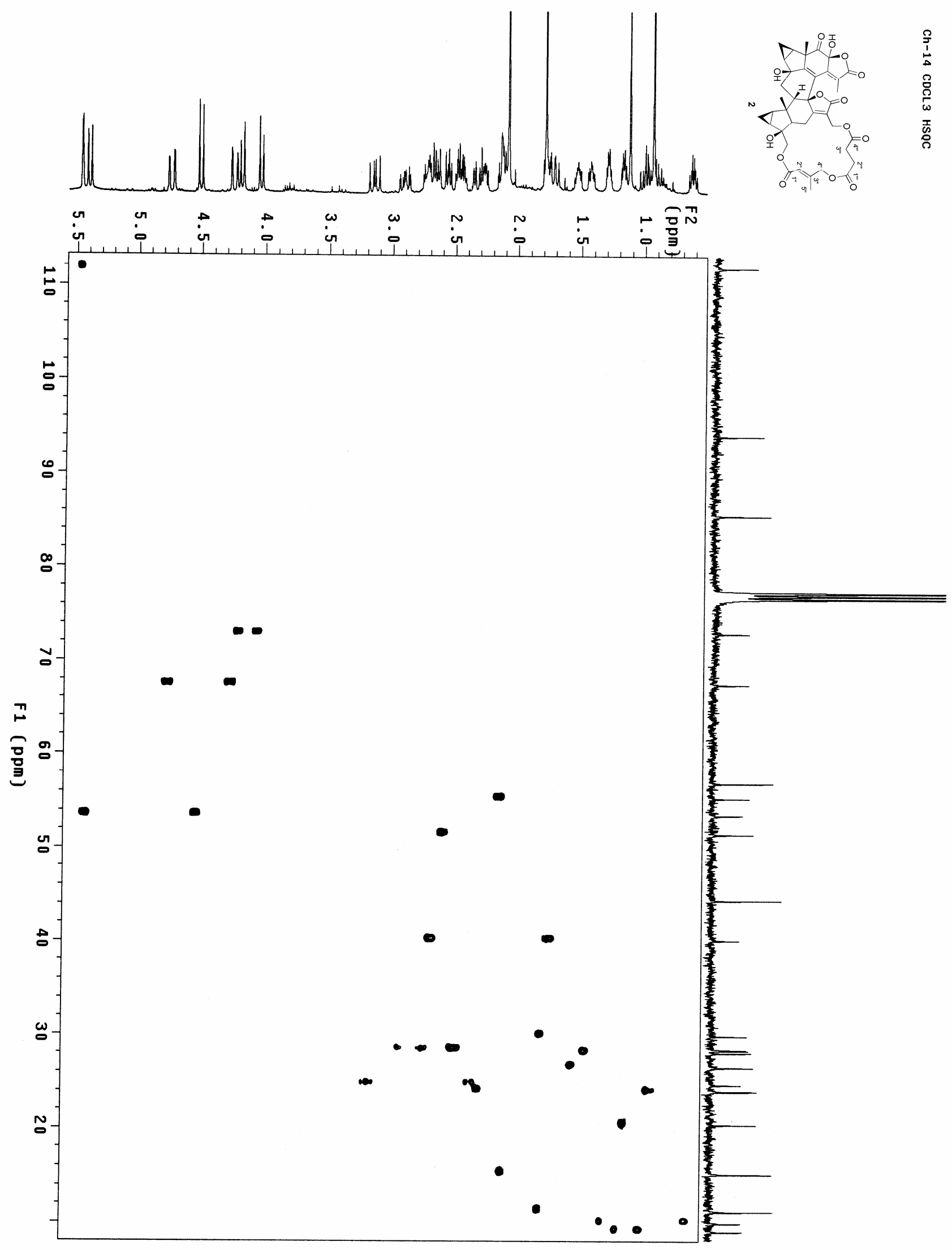

24 


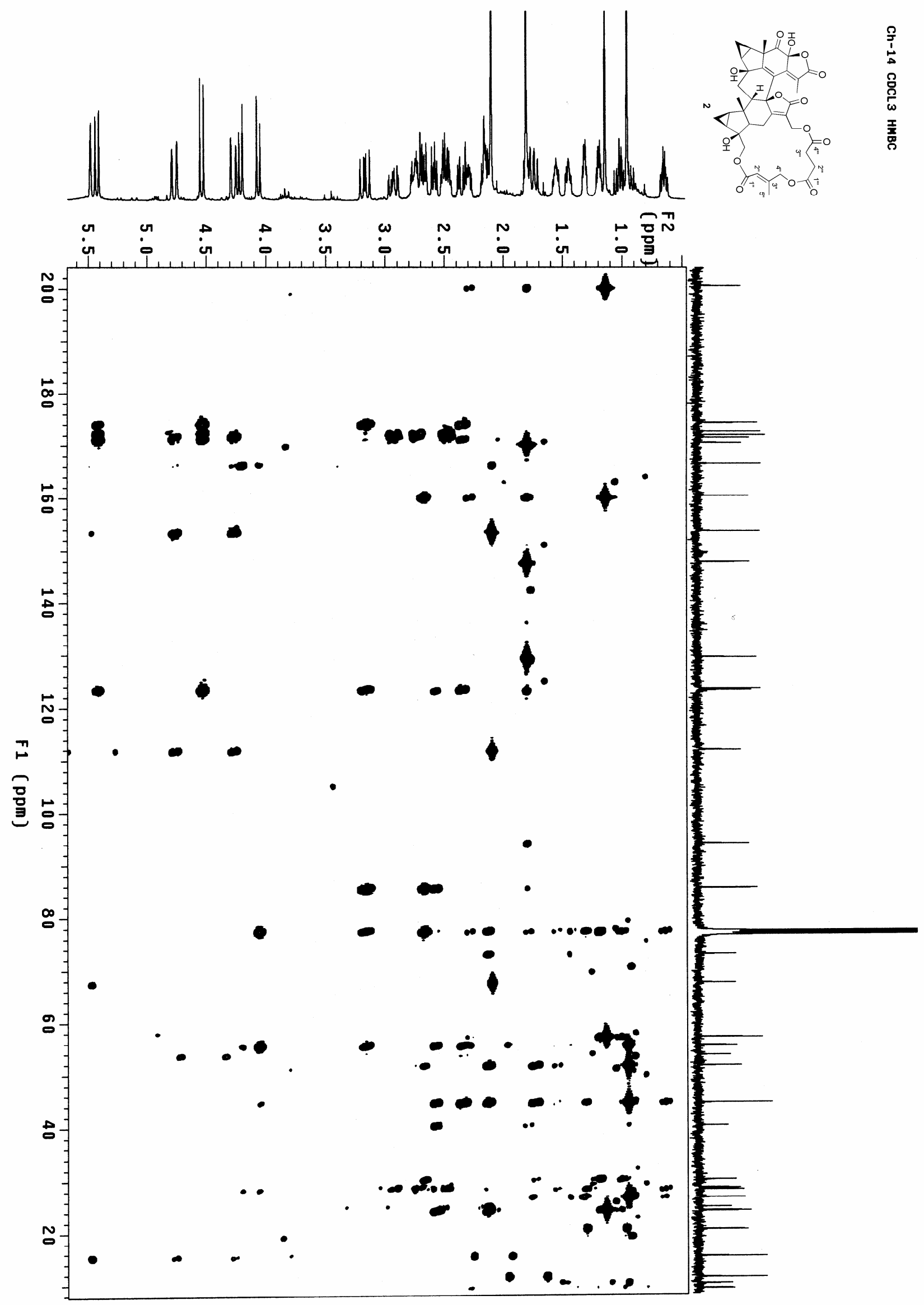




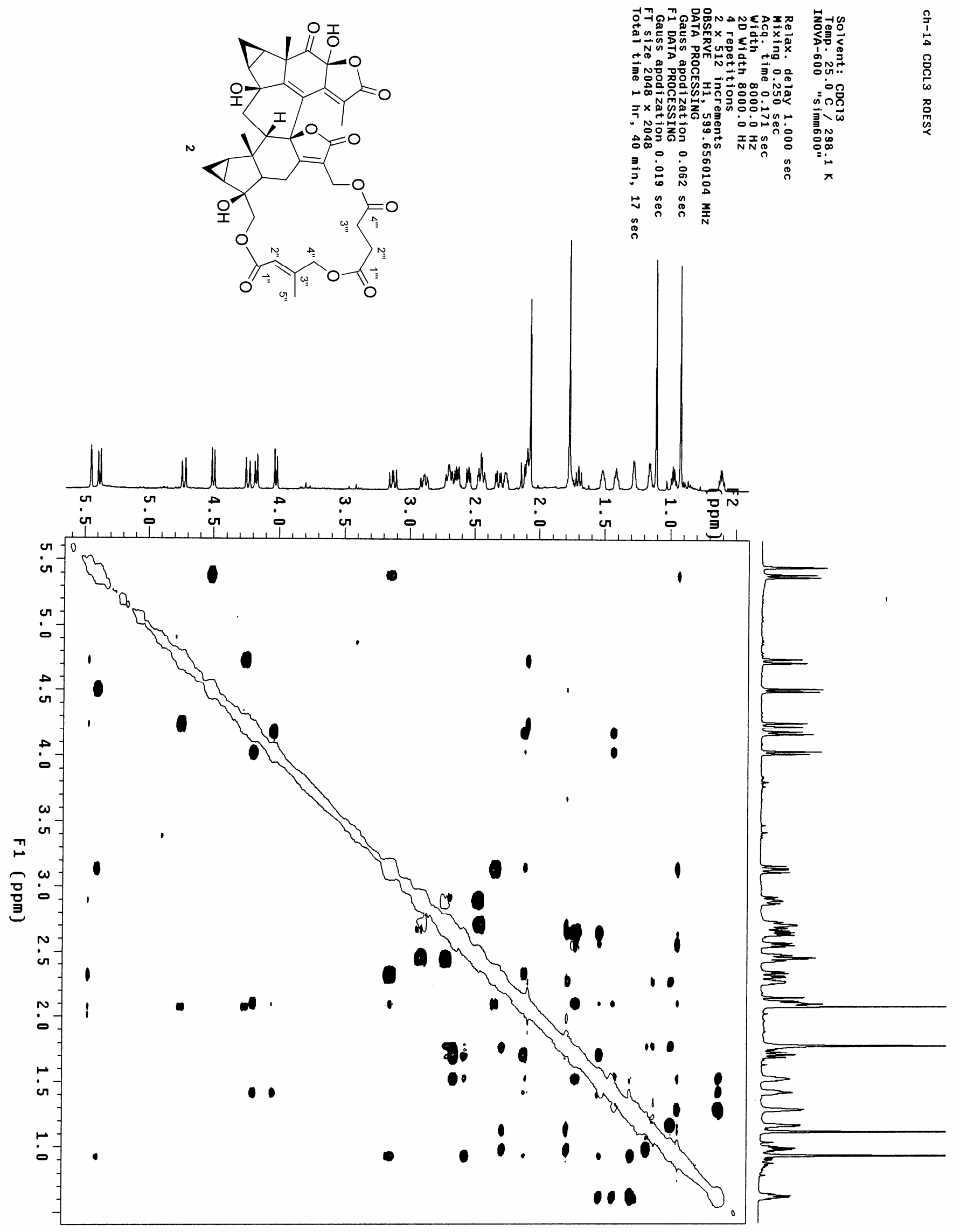


Figure S27. IR of chlorahololide B (2)

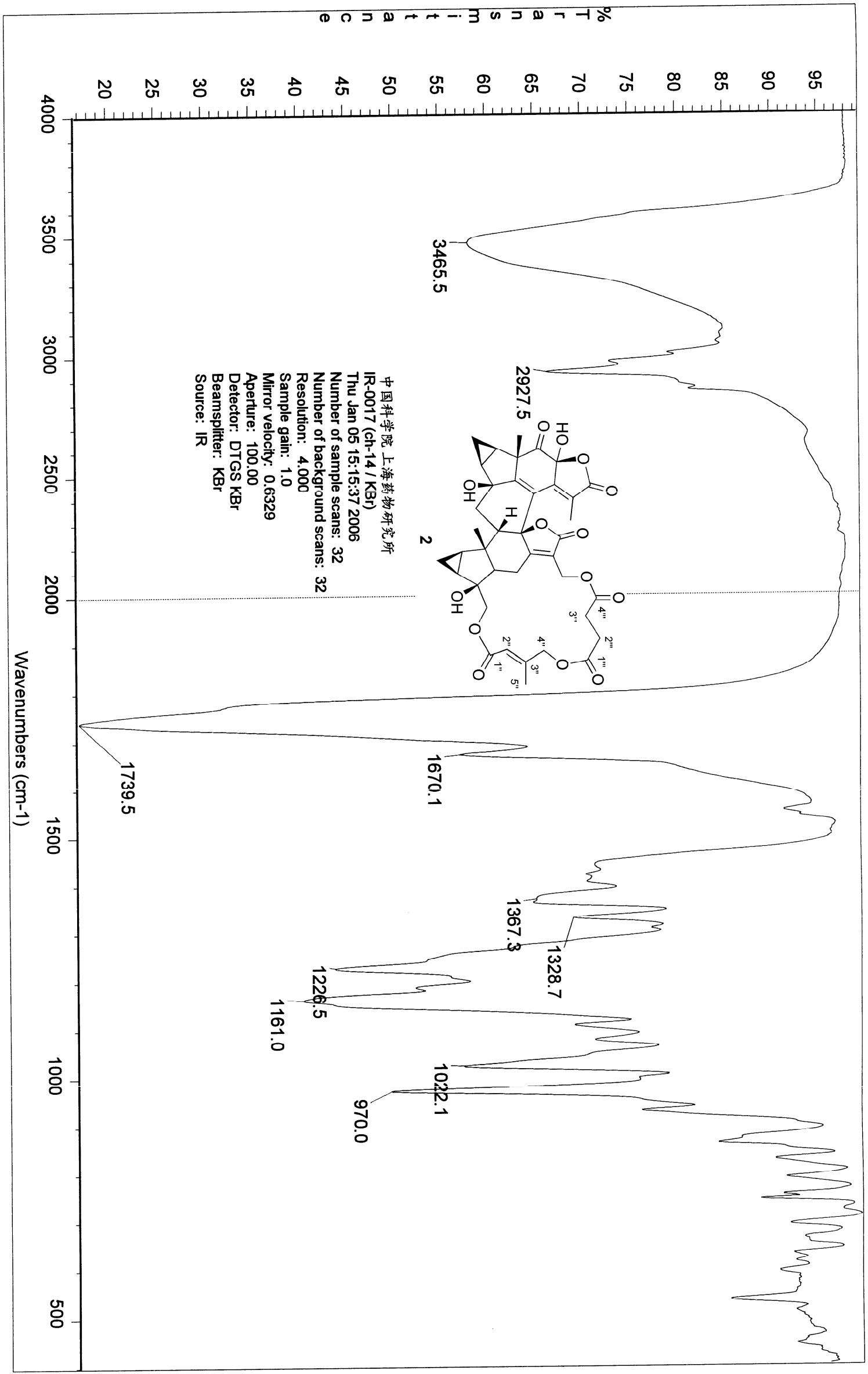


Figure S28. The atom serial numbers and molecular packing of chlorahololide A (1) in X-ray analysis

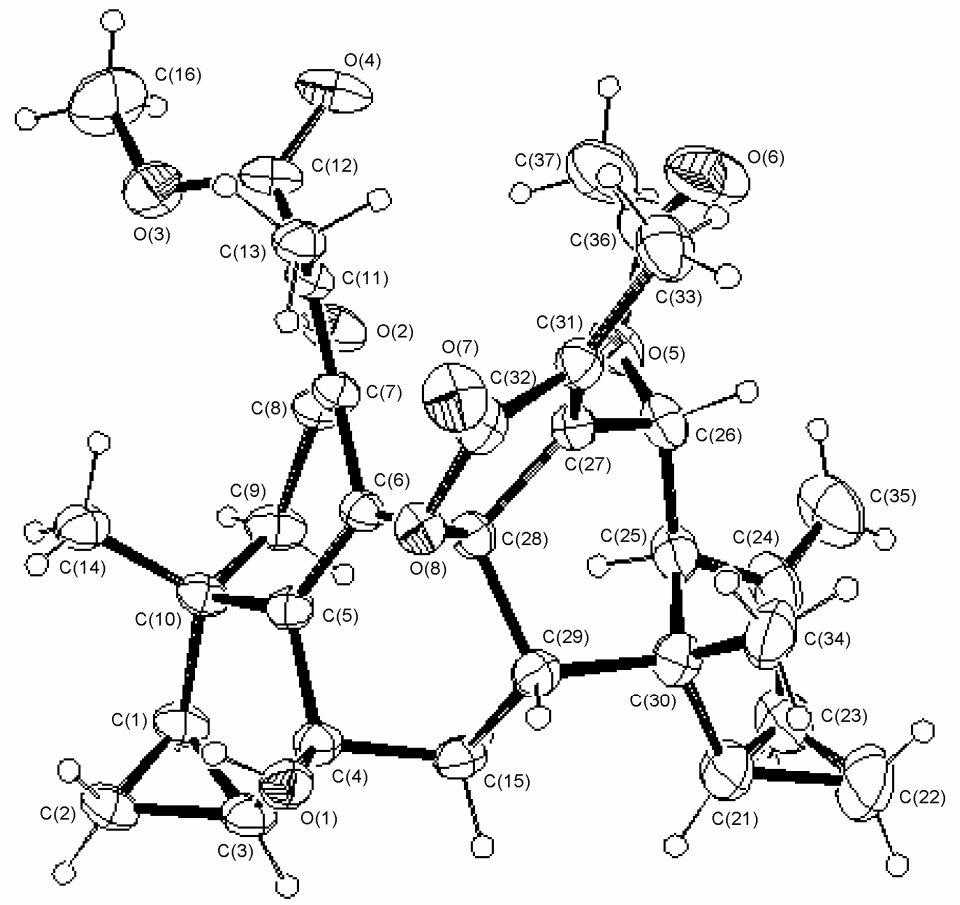

The molecular structure and the serial numbers in X-ray analysis of chlorahololide A (1).

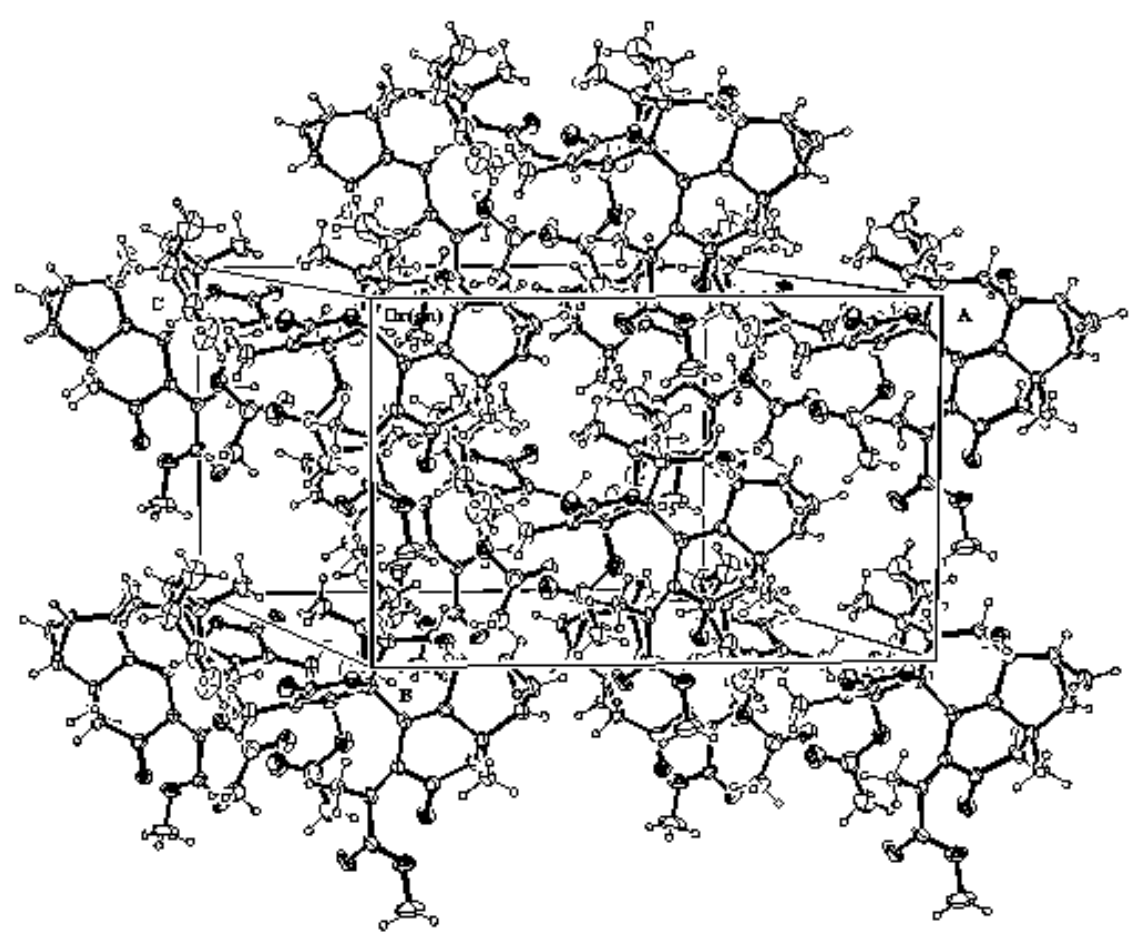

The molecular packing along the $c$ axis of chlorahololide A (1). 
Table 2. Crystal data and structure refinement for chlorahololide A (1) (labeled as ch2).

\begin{tabular}{|c|c|}
\hline Identification code & $\operatorname{ch} 2$ \\
\hline Empirical formula & C33 H36 O8 \\
\hline Formula weight & 560.62 \\
\hline Temperature & 293(2) K \\
\hline Wavelength & $0.71073 \mathrm{~A}$ \\
\hline Crystal system, space group & Monoclinic, C 2 \\
\hline \multirow[t]{3}{*}{ Unit cell dimensions } & $\mathrm{a}=17.216(6) \mathrm{A} \quad$ alpha $=90.00(4) \mathrm{deg}$ \\
\hline & $\mathrm{b}=11.078(6) \mathrm{A} \quad \mathrm{beta}=108.63(4) \mathrm{deg}$. \\
\hline & $\mathrm{c}=16.173(7) \mathrm{A} \quad$ gamma $=90.00(4) \mathrm{deg}$. \\
\hline Volume & $2923(2) A^{\wedge} 3$ \\
\hline Z, Calculated density & $4, \quad 1.274 \mathrm{Mg} / \mathrm{m}^{\wedge} 3$ \\
\hline Absorption coefficient & $0.091 \mathrm{~mm}^{\wedge}-1$ \\
\hline $\mathrm{F}(000)$ & 1192 \\
\hline Crystal size & $0.40 \times 0.35 \times 0.35 \mathrm{~mm}$ \\
\hline Theta range for data collection & 1.33 to $25.17 \mathrm{deg}$. \\
\hline Index ranges & $-20<=\mathrm{h}<=19,0<=\mathrm{k}<=13,-1<=\mathrm{l}<=19$ \\
\hline Reflections collected / unique & $3097 / 2782[\mathrm{R}(\mathrm{int})=0.0367]$ \\
\hline Completeness to 2 theta $=25.17$ & $99.9 \%$ \\
\hline Absorption correction & None \\
\hline Max. and min. transmission & 0.9690 and 0.9647 \\
\hline Refinement method & Full-matrix least-squares on $\mathrm{F}^{\wedge} 2$ \\
\hline Data / restraints / parameters & $2782 / 1 / 378$ \\
\hline Goodness-of-fit on $\mathrm{F}^{\wedge} 2$ & 1.085 \\
\hline Final $R$ indices $[I>2 \operatorname{sigma}(I)]$ & $\mathrm{R} 1=0.0366, \mathrm{wR} 2=0.0893$ \\
\hline $\mathrm{R}$ indices (all data) & $\mathrm{R} 1=0.0761, \mathrm{wR} 2=0.1039$ \\
\hline Absolute structure parameter & $0.2(15)$ \\
\hline Extinction coefficient & $0.0021(4)$ \\
\hline Largest diff. peak and hole & 0.148 and -0.171 e. $A^{\wedge}-3$ \\
\hline
\end{tabular}


Table 3. Atomic coordinates $\left(\times 10^{\wedge} 4\right)$ and equivalent isotropic displacement parameters $\left(\mathrm{A}^{\wedge} 2 \mathrm{x}\right.$ $10^{\wedge} 3$ ) for chlorahololide A (1) (labeled as ch2).

\begin{tabular}{|c|c|c|c|c|}
\hline & $\mathrm{x}$ & $\mathrm{y}$ & $\mathrm{z}$ & $\mathrm{U}(\mathrm{eq})$ \\
\hline $\mathrm{O}(1)$ & $8350(2)$ & $9543(2)$ & $8963(2)$ & $49(1)$ \\
\hline $\mathrm{O}(2)$ & $8075(2)$ & 14881(3) & $7215(2)$ & $64(1)$ \\
\hline $\mathrm{O}(3)$ & $9089(2)$ & $15752(3)$ & $8945(2)$ & $71(1)$ \\
\hline $\mathrm{O}(4)$ & $9800(2)$ & $15933(3)$ & $8003(2)$ & 77(1) \\
\hline $\mathrm{O}(5)$ & $9267(2)$ & $13007(3)$ & $6286(2)$ & $60(1)$ \\
\hline $\mathrm{O}(6)$ & $10495(2)$ & $13629(4)$ & $6279(3)$ & $110(1)$ \\
\hline $\mathrm{O}(7)$ & $11296(2)$ & $10661(3)$ & $9375(2)$ & 59(1) \\
\hline $\mathrm{O}(8)$ & 9935(1) & $10639(2)$ & $8753(2)$ & $44(1)$ \\
\hline $\mathrm{C}(1)$ & $6745(2)$ & $11658(4)$ & $8307(3)$ & $51(1)$ \\
\hline $\mathrm{C}(2)$ & $6820(2)$ & $10816(4)$ & $9044(3)$ & $58(1)$ \\
\hline $\mathrm{C}(3)$ & $6968(2)$ & $10353(4)$ & $8235(3)$ & $54(1)$ \\
\hline $\mathrm{C}(4)$ & $7870(2)$ & 10291(3) & $8283(2)$ & $44(1)$ \\
\hline$C(5)$ & $8132(2)$ & $11597(3)$ & $8334(2)$ & $39(1)$ \\
\hline$C(6)$ & $8792(2)$ & 11964(3) & $8126(2)$ & $38(1)$ \\
\hline$C(7)$ & $8882(2)$ & $13290(3)$ & $8055(2)$ & $39(1)$ \\
\hline $\mathrm{C}(8)$ & $8086(2)$ & $13916(4)$ & $7561(3)$ & $47(1)$ \\
\hline C(9) & $7318(2)$ & $13280(4)$ & $7573(3)$ & $56(1)$ \\
\hline$C(10)$ & $7469(2)$ & $12475(4)$ & $8380(2)$ & $44(1)$ \\
\hline $\mathrm{C}(11)$ & $9525(2)$ & $13985(3)$ & $8471(2)$ & $40(1)$ \\
\hline$C(12)$ & $9480(2)$ & $15325(4)$ & $8409(3)$ & $51(1)$ \\
\hline$C(13)$ & $10312(2)$ & $13555(4)$ & $9111(2)$ & $47(1)$ \\
\hline$C(14)$ & $7679(2)$ & $13263(4)$ & $9203(3)$ & $55(1)$ \\
\hline$C(15)$ & $7985(2)$ & $9790(4)$ & $7458(3)$ & 51(1) \\
\hline$C(16)$ & $8978(5)$ & $17046(5)$ & $8941(5)$ & $108(2)$ \\
\hline $\mathrm{C}(21)$ & $8274(3)$ & $8935(5)$ & $5899(3)$ & $75(2)$ \\
\hline$C(22)$ & $8410(4)$ & $8434(7)$ & $5096(4)$ & $110(2)$ \\
\hline $\mathrm{C}(23)$ & $7966(3)$ & 9591(7) & $5049(3)$ & $87(2)$ \\
\hline $\mathrm{C}(24)$ & $8452(3)$ & $10740(6)$ & $5137(3)$ & $76(2)$ \\
\hline $\mathrm{C}(25)$ & $8830(2)$ & $10949(5)$ & $6113(2)$ & $57(1)$ \\
\hline$C(26)$ & $9559(2)$ & $11778(4)$ & $6472(2)$ & $52(1)$ \\
\hline $\mathrm{C}(27)$ & $9901(2)$ & $11534(3)$ & $7432(2)$ & $42(1)$ \\
\hline $\mathrm{C}(28)$ & $9349(2)$ & $11017(3)$ & $7921(2)$ & $39(1)$ \\
\hline C(29) & $8874(2)$ & $9843(4)$ & $7466(2)$ & $46(1)$ \\
\hline$C(30)$ & $8960(3)$ & $9684(4)$ & $6542(3)$ & $59(1)$ \\
\hline $\mathrm{C}(31)$ & $10680(2)$ & $11456(4)$ & $7934(2)$ & $44(1)$ \\
\hline$C(32)$ & $10706(2)$ & $10897(4)$ & $8759(2)$ & $45(1)$ \\
\hline$C(33)$ & $11468(2)$ & $11793(5)$ & $7777(3)$ & $66(1)$ \\
\hline$C(34)$ & 9799(3) & $9093(5)$ & $6626(3)$ & 76(1) \\
\hline$C(35)$ & $8514(4)$ & $11442(7)$ & $4521(3)$ & $109(2)$ \\
\hline$C(36)$ & $9795(3)$ & $13848(5)$ & $6181(3)$ & 71(1) \\
\hline$C(37)$ & $9411(4)$ & $15035(5)$ & $5948(4)$ & $91(2)$ \\
\hline
\end{tabular}

$\mathrm{U}(\mathrm{eq})$ is defined as one third of the trace of the orthogonalized Uij tensor. 
Table 4. Bond lengths [A] and angles [deg] for chlorahololide A (1) (labeled as ch2).

\begin{tabular}{|c|c|}
\hline $\mathrm{O}(1)-\mathrm{C}(4)$ & $1.413(4)$ \\
\hline $\mathrm{O}(1)-\mathrm{H}(1)$ & 0.8200 \\
\hline $\mathrm{O}(2)-\mathrm{C}(8)$ & $1.204(5)$ \\
\hline $\mathrm{O}(3)-\mathrm{C}(12)$ & $1.342(5)$ \\
\hline $\mathrm{O}(3)-\mathrm{C}(16)$ & $1.446(6)$ \\
\hline $\mathrm{O}(4)-\mathrm{C}(12)$ & $1.190(5)$ \\
\hline $\mathrm{O}(5)-\mathrm{C}(36)$ & $1.350(6)$ \\
\hline $\mathrm{O}(5)-\mathrm{C}(26)$ & $1.449(5)$ \\
\hline $\mathrm{O}(6)-\mathrm{C}(36)$ & $1.189(5)$ \\
\hline $\mathrm{O}(7)-\mathrm{C}(32)$ & $1.203(4)$ \\
\hline $\mathrm{O}(8)-\mathrm{C}(32)$ & $1.354(4)$ \\
\hline $\mathrm{O}(8)-\mathrm{C}(28)$ & $1.462(4)$ \\
\hline$C(1)-C(2)$ & $1.487(6)$ \\
\hline C(1)-C(3) & $1.509(6)$ \\
\hline C(1)-C(10) & $1.514(5)$ \\
\hline $\mathrm{C}(1)-\mathrm{H}(1 \mathrm{~A})$ & 0.9800 \\
\hline $\mathrm{C}(2)-\mathrm{C}(3)$ & $1.502(6)$ \\
\hline $\mathrm{C}(2)-\mathrm{H}(2 \mathrm{~A})$ & 0.9700 \\
\hline $\mathrm{C}(2)-\mathrm{H}(2 \mathrm{~B})$ & 0.9700 \\
\hline $\mathrm{C}(3)-\mathrm{C}(4)$ & $1.531(5)$ \\
\hline $\mathrm{C}(3)-\mathrm{H}(3)$ & 0.9800 \\
\hline$C(4)-C(5)$ & $1.509(5)$ \\
\hline$C(4)-C(15)$ & $1.515(5)$ \\
\hline$C(5)-C(6)$ & $1.346(5)$ \\
\hline$C(5)-C(10)$ & $1.520(5)$ \\
\hline $\mathrm{C}(6)-\mathrm{C}(7)$ & $1.486(5)$ \\
\hline$C(6)-C(28)$ & $1.528(5)$ \\
\hline$C(7)-C(11)$ & $1.340(5)$ \\
\hline$C(7)-C(8)$ & $1.515(5)$ \\
\hline $\mathrm{C}(8)-\mathrm{C}(9)$ & $1.505(5)$ \\
\hline$C(9)-C(10)$ & $1.532(6)$ \\
\hline $\mathrm{C}(9)-\mathrm{H}(9 \mathrm{~A})$ & 0.9700 \\
\hline $\mathrm{C}(9)-\mathrm{H}(9 \mathrm{~B})$ & 0.9700 \\
\hline$C(10)-C(14)$ & $1.534(6)$ \\
\hline C(11)-C(12) & $1.488(6)$ \\
\hline C(11)-C(13) & $1.497(5)$ \\
\hline $\mathrm{C}(13)-\mathrm{H}(13 \mathrm{~A})$ & 0.9599 \\
\hline $\mathrm{C}(13)-\mathrm{H}(13 \mathrm{~B})$ & 0.9599 \\
\hline $\mathrm{C}(13)-\mathrm{H}(13 \mathrm{C})$ & 0.9599 \\
\hline $\mathrm{C}(14)-\mathrm{H}(14 \mathrm{~A})$ & 0.9599 \\
\hline $\mathrm{C}(14)-\mathrm{H}(14 \mathrm{~B})$ & 0.9599 \\
\hline $\mathrm{C}(14)-\mathrm{H}(14 \mathrm{C})$ & 0.9599 \\
\hline C(15)-C(29) & $1.529(5)$ \\
\hline $\mathrm{C}(15)-\mathrm{H}(15 \mathrm{~A})$ & 0.9700 \\
\hline $\mathrm{C}(15)-\mathrm{H}(15 \mathrm{~B})$ & 0.9700 \\
\hline $\mathrm{C}(16)-\mathrm{H}(16 \mathrm{~A})$ & 0.9599 \\
\hline $\mathrm{C}(16)-\mathrm{H}(16 \mathrm{~B})$ & 0.9599 \\
\hline $\mathrm{C}(16)-\mathrm{H}(16 \mathrm{C})$ & 0.9599 \\
\hline $\mathrm{C}(21)-\mathrm{C}(23)$ & $1.494(8)$ \\
\hline$C(21)-C(22)$ & $1.497(7)$ \\
\hline$C(21)-C(30)$ & $1.542(6)$ \\
\hline $\mathrm{C}(21)-\mathrm{H}(21)$ & 0.9800 \\
\hline $\mathrm{C}(22)-\mathrm{C}(23)$ & $1.482(10)$ \\
\hline $\mathrm{C}(22)-\mathrm{H}(22 \mathrm{~A})$ & 0.9700 \\
\hline $\mathrm{C}(22)-\mathrm{H}(22 \mathrm{~B})$ & 0.9700 \\
\hline$C(23)-C(24)$ & $1.505(9)$ \\
\hline $\mathrm{C}(23)-\mathrm{H}(23)$ & 0.9800 \\
\hline$C(24)-C(35)$ & $1.294(8)$ \\
\hline
\end{tabular}




\begin{tabular}{|c|c|}
\hline$C(24)-C(25)$ & $1.520(6)$ \\
\hline$C(25)-C(26)$ & $1.513(6)$ \\
\hline$C(25)-C(30)$ & $1.548(7)$ \\
\hline $\mathrm{C}(25)-\mathrm{H}(25)$ & 0.9800 \\
\hline$C(26)-C(27)$ & $1.499(5)$ \\
\hline $\mathrm{C}(26)-\mathrm{H}(26)$ & 0.9800 \\
\hline$C(27)-C(31)$ & $1.331(5)$ \\
\hline$C(27)-C(28)$ & $1.529(5)$ \\
\hline$C(28)-C(29)$ & $1.586(5)$ \\
\hline C(29)-C(30) & $1.558(6)$ \\
\hline $\mathrm{C}(29)-\mathrm{H}(29)$ & 0.9800 \\
\hline $\mathrm{C}(30)-\mathrm{C}(34)$ & $1.552(6)$ \\
\hline $\mathrm{C}(31)-\mathrm{C}(32)$ & $1.458(5)$ \\
\hline C(31)-C(33) & $1.504(5)$ \\
\hline $\mathrm{C}(33)-\mathrm{H}(33 \mathrm{~A})$ & 0.9599 \\
\hline $\mathrm{C}(33)-\mathrm{H}(33 \mathrm{~B})$ & 0.9599 \\
\hline $\mathrm{C}(33)-\mathrm{H}(33 \mathrm{C})$ & 0.9599 \\
\hline $\mathrm{C}(34)-\mathrm{H}(34 \mathrm{~A})$ & 0.9599 \\
\hline $\mathrm{C}(34)-\mathrm{H}(34 \mathrm{~B})$ & 0.9599 \\
\hline $\mathrm{C}(34)-\mathrm{H}(34 \mathrm{C})$ & 0.9599 \\
\hline $\mathrm{C}(35)-\mathrm{H}(35 \mathrm{~A})$ & 0.9300 \\
\hline $\mathrm{C}(35)-\mathrm{H}(35 \mathrm{~B})$ & 0.9300 \\
\hline $\mathrm{C}(36)-\mathrm{C}(37)$ & $1.467(7)$ \\
\hline $\mathrm{C}(37)-\mathrm{H}(37 \mathrm{~A})$ & 0.9599 \\
\hline $\mathrm{C}(37)-\mathrm{H}(37 \mathrm{~B})$ & 0.9599 \\
\hline $\mathrm{C}(37)-\mathrm{H}(37 \mathrm{C})$ & 0.9599 \\
\hline $\mathrm{C}(4)-\mathrm{O}(1)-\mathrm{H}(1)$ & 109.5 \\
\hline $\mathrm{C}(12)-\mathrm{O}(3)-\mathrm{C}(16)$ & $116.1(4)$ \\
\hline $\mathrm{C}(36)-\mathrm{O}(5)-\mathrm{C}(26)$ & $118.1(3)$ \\
\hline $\mathrm{C}(32)-\mathrm{O}(8)-\mathrm{C}(28)$ & $109.4(3)$ \\
\hline C(2)-C(1)-C(3) & 60.1(3) \\
\hline $\mathrm{C}(2)-\mathrm{C}(1)-\mathrm{C}(10)$ & $117.0(3)$ \\
\hline C(3)-C(1)-C(10) & $110.8(3)$ \\
\hline $\mathrm{C}(2)-\mathrm{C}(1)-\mathrm{H}(1 \mathrm{~A})$ & 118.4 \\
\hline C(3)-C(1)-H(1A) & 118.4 \\
\hline $\mathrm{C}(10)-\mathrm{C}(1)-\mathrm{H}(1 \mathrm{~A})$ & 118.4 \\
\hline $\mathrm{C}(1)-\mathrm{C}(2)-\mathrm{C}(3)$ & $60.7(3)$ \\
\hline $\mathrm{C}(1)-\mathrm{C}(2)-\mathrm{H}(2 \mathrm{~A})$ & 117.7 \\
\hline $\mathrm{C}(3)-\mathrm{C}(2)-\mathrm{H}(2 \mathrm{~A})$ & 117.7 \\
\hline $\mathrm{C}(1)-\mathrm{C}(2)-\mathrm{H}(2 \mathrm{~B})$ & 117.7 \\
\hline $\mathrm{C}(3)-\mathrm{C}(2)-\mathrm{H}(2 \mathrm{~B})$ & 117.7 \\
\hline $\mathrm{H}(2 \mathrm{~A})-\mathrm{C}(2)-\mathrm{H}(2 \mathrm{~B})$ & 114.8 \\
\hline$C(2)-C(3)-C(1)$ & $59.2(3)$ \\
\hline$C(2)-C(3)-C(4)$ & $115.0(3)$ \\
\hline $\mathrm{C}(1)-\mathrm{C}(3)-\mathrm{C}(4)$ & $108.5(3)$ \\
\hline $\mathrm{C}(2)-\mathrm{C}(3)-\mathrm{H}(3)$ & 119.7 \\
\hline $\mathrm{C}(1)-\mathrm{C}(3)-\mathrm{H}(3)$ & 119.7 \\
\hline $\mathrm{C}(4)-\mathrm{C}(3)-\mathrm{H}(3)$ & 119.7 \\
\hline $\mathrm{O}(1)-\mathrm{C}(4)-\mathrm{C}(5)$ & $115.5(3)$ \\
\hline $\mathrm{O}(1)-\mathrm{C}(4)-\mathrm{C}(15)$ & $105.2(3)$ \\
\hline C(5)-C(4)-C(15) & 106.1(3) \\
\hline $\mathrm{O}(1)-\mathrm{C}(4)-\mathrm{C}(3)$ & $113.4(3)$ \\
\hline C(5)-C(4)-C(3) & $103.9(3)$ \\
\hline $\mathrm{C}(15)-\mathrm{C}(4)-\mathrm{C}(3)$ & $112.7(3)$ \\
\hline C(6)-C(5)-C(4) & $122.5(3)$ \\
\hline $\mathrm{C}(6)-\mathrm{C}(5)-\mathrm{C}(10)$ & $121.7(3)$ \\
\hline $\mathrm{C}(4)-\mathrm{C}(5)-\mathrm{C}(10)$ & $113.6(3)$ \\
\hline $\mathrm{C}(5)-\mathrm{C}(6)-\mathrm{C}(7)$ & $115.8(3)$ \\
\hline C(5)-C(6)-C(28) & $119.0(3)$ \\
\hline$C(7)-C(6)-C(28)$ & $125.1(3)$ \\
\hline $\mathrm{C}(11)-\mathrm{C}(7)-\mathrm{C}(6)$ & $128.3(3)$ \\
\hline $\mathrm{C}(11)-\mathrm{C}(7)-\mathrm{C}(8)$ & $117.7(3)$ \\
\hline
\end{tabular}




\begin{tabular}{|c|c|}
\hline$C(6)-C(7)-C(8)$ & $113.2(3)$ \\
\hline $\mathrm{O}(2)-\mathrm{C}(8)-\mathrm{C}(9)$ & $122.6(4)$ \\
\hline $\mathrm{O}(2)-\mathrm{C}(8)-\mathrm{C}(7)$ & $121.9(4)$ \\
\hline $\mathrm{C}(9)-\mathrm{C}(8)-\mathrm{C}(7)$ & $115.4(3)$ \\
\hline $\mathrm{C}(8)-\mathrm{C}(9)-\mathrm{C}(10)$ & $111.9(3)$ \\
\hline $\mathrm{C}(8)-\mathrm{C}(9)-\mathrm{H}(9 \mathrm{~A})$ & 109.2 \\
\hline $\mathrm{C}(10)-\mathrm{C}(9)-\mathrm{H}(9 \mathrm{~A})$ & 109.2 \\
\hline C(8)-C(9)-H(9B) & 109.2 \\
\hline $\mathrm{C}(10)-\mathrm{C}(9)-\mathrm{H}(9 \mathrm{~B})$ & 109.2 \\
\hline $\mathrm{H}(9 \mathrm{~A})-\mathrm{C}(9)-\mathrm{H}(9 \mathrm{~B})$ & 107.9 \\
\hline$C(1)-C(10)-C(5)$ & $103.0(3)$ \\
\hline $\mathrm{C}(1)-\mathrm{C}(10)-\mathrm{C}(9)$ & $111.7(3)$ \\
\hline $\mathrm{C}(5)-\mathrm{C}(10)-\mathrm{C}(9)$ & $104.9(3)$ \\
\hline $\mathrm{C}(1)-\mathrm{C}(10)-\mathrm{C}(14)$ & $111.6(3)$ \\
\hline$C(5)-C(10)-C(14)$ & $115.8(3)$ \\
\hline $\mathrm{C}(9)-\mathrm{C}(10)-\mathrm{C}(14)$ & $109.6(3)$ \\
\hline $\mathrm{C}(7)-\mathrm{C}(11)-\mathrm{C}(12)$ & $121.5(3)$ \\
\hline C(7)-C(11)-C(13) & $125.8(3)$ \\
\hline $\mathrm{C}(12)-\mathrm{C}(11)-\mathrm{C}(13)$ & $112.3(3)$ \\
\hline $\mathrm{O}(4)-\mathrm{C}(12)-\mathrm{O}(3)$ & $124.9(4)$ \\
\hline $\mathrm{O}(4)-\mathrm{C}(12)-\mathrm{C}(11)$ & $125.3(4)$ \\
\hline $\mathrm{O}(3)-\mathrm{C}(12)-\mathrm{C}(11)$ & $109.5(4)$ \\
\hline $\mathrm{C}(11)-\mathrm{C}(13)-\mathrm{H}(13 \mathrm{~A})$ & 109.5 \\
\hline $\mathrm{C}(11)-\mathrm{C}(13)-\mathrm{H}(13 \mathrm{~B})$ & 109.5 \\
\hline $\mathrm{H}(13 \mathrm{~A})-\mathrm{C}(13)-\mathrm{H}(13 \mathrm{~B})$ & 109.5 \\
\hline C(11)-C(13)-H(13C) & 109.5 \\
\hline $\mathrm{H}(13 \mathrm{~A})-\mathrm{C}(13)-\mathrm{H}(13 \mathrm{C})$ & 109.5 \\
\hline $\mathrm{H}(13 \mathrm{~B})-\mathrm{C}(13)-\mathrm{H}(13 \mathrm{C})$ & 109.5 \\
\hline C(10)-C(14)-H(14A) & 109.5 \\
\hline C(10)-C(14)-H(14B) & 109.5 \\
\hline $\mathrm{H}(14 \mathrm{~A})-\mathrm{C}(14)-\mathrm{H}(14 \mathrm{~B})$ & 109.5 \\
\hline $\mathrm{C}(10)-\mathrm{C}(14)-\mathrm{H}(14 \mathrm{C})$ & 109.5 \\
\hline $\mathrm{H}(14 \mathrm{~A})-\mathrm{C}(14)-\mathrm{H}(14 \mathrm{C})$ & 109.5 \\
\hline $\mathrm{H}(14 \mathrm{~B})-\mathrm{C}(14)-\mathrm{H}(14 \mathrm{C})$ & 109.5 \\
\hline $\mathrm{C}(4)-\mathrm{C}(15)-\mathrm{C}(29)$ & $113.0(3)$ \\
\hline $\mathrm{C}(4)-\mathrm{C}(15)-\mathrm{H}(15 \mathrm{~A})$ & 109.0 \\
\hline $\mathrm{C}(29)-\mathrm{C}(15)-\mathrm{H}(15 \mathrm{~A})$ & 109.0 \\
\hline $\mathrm{C}(4)-\mathrm{C}(15)-\mathrm{H}(15 \mathrm{~B})$ & 109.0 \\
\hline C(29)-C(15)-H(15B) & 109.0 \\
\hline $\mathrm{H}(15 \mathrm{~A})-\mathrm{C}(15)-\mathrm{H}(15 \mathrm{~B})$ & 107.8 \\
\hline $\mathrm{O}(3)-\mathrm{C}(16)-\mathrm{H}(16 \mathrm{~A})$ & 109.5 \\
\hline $\mathrm{O}(3)-\mathrm{C}(16)-\mathrm{H}(16 \mathrm{~B})$ & 109.5 \\
\hline $\mathrm{H}(16 \mathrm{~A})-\mathrm{C}(16)-\mathrm{H}(16 \mathrm{~B})$ & 109.5 \\
\hline $\mathrm{O}(3)-\mathrm{C}(16)-\mathrm{H}(16 \mathrm{C})$ & 109.5 \\
\hline $\mathrm{H}(16 \mathrm{~A})-\mathrm{C}(16)-\mathrm{H}(16 \mathrm{C})$ & 109.5 \\
\hline $\mathrm{H}(16 \mathrm{~B})-\mathrm{C}(16)-\mathrm{H}(16 \mathrm{C})$ & 109.5 \\
\hline $\mathrm{C}(23)-\mathrm{C}(21)-\mathrm{C}(22)$ & $59.4(4)$ \\
\hline $\mathrm{C}(23)-\mathrm{C}(21)-\mathrm{C}(30)$ & $109.2(4)$ \\
\hline$C(22)-C(21)-C(30)$ & $119.0(4)$ \\
\hline $\mathrm{C}(23)-\mathrm{C}(21)-\mathrm{H}(21)$ & 118.3 \\
\hline $\mathrm{C}(22)-\mathrm{C}(21)-\mathrm{H}(21)$ & 118.3 \\
\hline $\mathrm{C}(30)-\mathrm{C}(21)-\mathrm{H}(21)$ & 118.3 \\
\hline $\mathrm{C}(23)-\mathrm{C}(22)-\mathrm{C}(21)$ & $60.2(4)$ \\
\hline $\mathrm{C}(23)-\mathrm{C}(22)-\mathrm{H}(22 \mathrm{~A})$ & 117.7 \\
\hline $\mathrm{C}(21)-\mathrm{C}(22)-\mathrm{H}(22 \mathrm{~A})$ & 117.7 \\
\hline $\mathrm{C}(23)-\mathrm{C}(22)-\mathrm{H}(22 \mathrm{~B})$ & 117.7 \\
\hline $\mathrm{C}(21)-\mathrm{C}(22)-\mathrm{H}(22 \mathrm{~B})$ & 117.7 \\
\hline $\mathrm{H}(22 \mathrm{~A})-\mathrm{C}(22)-\mathrm{H}(22 \mathrm{~B})$ & 114.9 \\
\hline $\mathrm{C}(22)-\mathrm{C}(23)-\mathrm{C}(21)$ & $60.4(4)$ \\
\hline $\mathrm{C}(22)-\mathrm{C}(23)-\mathrm{C}(24)$ & $117.7(5)$ \\
\hline $\mathrm{C}(21)-\mathrm{C}(23)-\mathrm{C}(24)$ & $107.5(4)$ \\
\hline $\mathrm{C}(22)-\mathrm{C}(23)-\mathrm{H}(23)$ & 118.9 \\
\hline $\mathrm{C}(21)-\mathrm{C}(23)-\mathrm{H}(23)$ & 118.9 \\
\hline
\end{tabular}


$\mathrm{H}(37 \mathrm{~A})-\mathrm{C}(37)-\mathrm{H}(37 \mathrm{~B})$

$\mathrm{C}(36)-\mathrm{C}(37)-\mathrm{H}(37 \mathrm{C})$

$\mathrm{H}(37 \mathrm{~A})-\mathrm{C}(37)-\mathrm{H}(37 \mathrm{C})$

$\mathrm{H}(37 \mathrm{~B})-\mathrm{C}(37)-\mathrm{H}(37 \mathrm{C})$
109.5

109.5

109.5

109.5

Symmetry transformations used to generate equivalent atoms: 
Table 5. Anisotropic displacement parameters $\left(\mathrm{A}^{\wedge} 2 \times 10^{\wedge} 3\right)$ for chlorahololide $\mathrm{A}(\mathbf{1})$ (labeled as $\left.\mathrm{ch} 2\right)$.

\begin{tabular}{|c|c|c|c|c|c|c|c|}
\hline & U11 & U22 & U33 & U23 & & U13 & U12 \\
\hline $\mathrm{O}(1)$ & $60(2)$ & $38(2)$ & $51(2)$ & $2(1)$ & $19(1)$ & $-1(1)$ & \\
\hline $\mathrm{O}(2)$ & $50(2)$ & $54(2)$ & $83(2)$ & $30(2)$ & $15(1)$ & $3(1)$ & \\
\hline $\mathrm{O}(3)$ & $89(2)$ & $38(2)$ & $90(2)$ & $2(2)$ & $36(2)$ & $8(2)$ & \\
\hline $\mathrm{O}(4)$ & $57(2)$ & $63(2)$ & $106(2)$ & $24(2)$ & $22(2)$ & $-19(2)$ & \\
\hline $\mathrm{O}(5)$ & $56(2)$ & $70(2)$ & $59(2)$ & $20(2)$ & $25(1)$ & $7(2)$ & \\
\hline $\mathrm{O}(6)$ & $70(2)$ & $86(3)$ & $185(4)$ & $14(3)$ & $55(3)$ & $-3(2)$ & \\
\hline $\mathrm{O}(7)$ & $40(1)$ & $75(2)$ & $55(2)$ & $7(2)$ & $6(1)$ & $12(2)$ & \\
\hline $\mathrm{O}(8)$ & $40(1)$ & $48(2)$ & $42(1)$ & $3(1)$ & $12(1)$ & $2(1)$ & \\
\hline $\mathrm{C}(1)$ & $29(2)$ & $54(3)$ & $67(3)$ & $4(2)$ & $14(2)$ & $-5(2)$ & \\
\hline $\mathrm{C}(2)$ & $39(2)$ & $65(3)$ & $75(3)$ & $5(3)$ & $27(2)$ & $-11(2)$ & \\
\hline $\mathrm{C}(3)$ & $39(2)$ & $58(3)$ & $63(3)$ & $-3(2)$ & $16(2)$ & $-18(2)$ & \\
\hline $\mathrm{C}(4)$ & $42(2)$ & $44(2)$ & $44(2)$ & $-2(2)$ & $12(2)$ & $-9(2)$ & \\
\hline$C(5)$ & $33(2)$ & $42(2)$ & $40(2)$ & $-1(2)$ & $7(2)$ & $-3(2)$ & \\
\hline C(6) & $31(2)$ & $43(2)$ & $38(2)$ & 1(2) & $10(2)$ & $-5(2)$ & \\
\hline$C(7)$ & $32(2)$ & $40(2)$ & $46(2)$ & $5(2)$ & $14(2)$ & $0(2)$ & \\
\hline $\mathrm{C}(8)$ & $38(2)$ & $45(2)$ & $56(2)$ & $5(2)$ & $11(2)$ & $-2(2)$ & \\
\hline $\mathrm{C}(9)$ & $32(2)$ & $54(3)$ & $75(3)$ & $10(2)$ & $9(2)$ & $-2(2)$ & \\
\hline$C(10)$ & $29(2)$ & $45(2)$ & $57(2)$ & $7(2)$ & $12(2)$ & $-1(2)$ & \\
\hline $\mathrm{C}(11)$ & $35(2)$ & $38(2)$ & $52(2)$ & $6(2)$ & $19(2)$ & $-2(2)$ & \\
\hline$C(12)$ & $37(2)$ & $42(2)$ & $69(3)$ & $10(2)$ & $9(2)$ & $-10(2)$ & \\
\hline$C(13)$ & $37(2)$ & $45(2)$ & $60(2)$ & $-3(2)$ & $18(2)$ & $-5(2)$ & \\
\hline$C(14)$ & $42(2)$ & $51(3)$ & $75(3)$ & $-6(2)$ & $23(2)$ & $-4(2)$ & \\
\hline$C(15)$ & $53(2)$ & $46(2)$ & $52(2)$ & $-8(2)$ & $14(2)$ & $-17(2)$ & \\
\hline$C(16)$ & $135(5)$ & $43(3)$ & $147(6)$ & $-6(3)$ & $44(4)$ & 14(3) & \\
\hline$C(21)$ & $76(3)$ & $94(4)$ & $56(3)$ & $-25(3)$ & $24(2)$ & $-26(3)$ & \\
\hline $\mathrm{C}(22)$ & $112(5)$ & $141(7)$ & $81(4)$ & $-56(4)$ & $37(4)$ & $-35(5)$ & \\
\hline $\mathrm{C}(23)$ & $74(3)$ & $132(6)$ & $53(3)$ & $-21(3)$ & $18(2)$ & $-31(4)$ & \\
\hline $\mathrm{C}(24)$ & $62(3)$ & $125(5)$ & $40(2)$ & $-1(3)$ & $16(2)$ & $-13(3)$ & \\
\hline$C(25)$ & $45(2)$ & $84(3)$ & $45(2)$ & $-6(2)$ & $16(2)$ & $-4(2)$ & \\
\hline$C(26)$ & $43(2)$ & $69(3)$ & $46(2)$ & $4(2)$ & $19(2)$ & $4(2)$ & \\
\hline $\mathrm{C}(27)$ & $41(2)$ & $42(2)$ & $44(2)$ & $-2(2)$ & $18(2)$ & $1(2)$ & \\
\hline $\mathrm{C}(28)$ & $39(2)$ & $42(2)$ & $35(2)$ & $4(2)$ & $12(2)$ & $2(2)$ & \\
\hline C(29) & $52(2)$ & $41(2)$ & $47(2)$ & $-3(2)$ & $16(2)$ & $-2(2)$ & \\
\hline$C(30)$ & $65(3)$ & $64(3)$ & $50(2)$ & $-9(2)$ & $22(2)$ & $-7(2)$ & \\
\hline$C(31)$ & $38(2)$ & $49(2)$ & $47(2)$ & $-5(2)$ & $15(2)$ & 1(2) & \\
\hline$C(32)$ & $39(2)$ & $45(2)$ & $48(2)$ & $-3(2)$ & $11(2)$ & $13(2)$ & \\
\hline C(33) & $42(2)$ & $90(4)$ & $69(3)$ & $-3(3)$ & $23(2)$ & $5(2)$ & \\
\hline$C(34)$ & $75(3)$ & $79(4)$ & $78(3)$ & $-28(3)$ & $32(3)$ & $4(3)$ & \\
\hline$C(35)$ & $106(4)$ & $174(7)$ & $45(3)$ & 1(4) & $22(3)$ & $-34(5)$ & \\
\hline$C(36)$ & $66(3)$ & 73(3) & $79(3)$ & $12(3)$ & $32(3)$ & 1(3) & \\
\hline $\mathrm{C}(37)$ & $93(4)$ & $82(4)$ & $105(4)$ & $34(3)$ & $41(3)$ & $8(3)$ & \\
\hline
\end{tabular}

The anisotropic displacement factor exponent takes the form: $-2 \mathrm{pi}^{\wedge} 2\left[\mathrm{~h}^{\wedge} 2 \mathrm{a}^{* \wedge} 2 \mathrm{U} 11+\ldots+2 \mathrm{~h} \mathrm{k} \mathrm{a} \mathrm{b}^{*} \mathrm{U} 12\right.$ ] 
Table 6. Hydrogen coordinates $\left(\mathrm{x} 10^{\wedge} 4\right)$ and isotropic displacement parameters $\left(\mathrm{A}^{\wedge} 2 \mathrm{x}\right.$ $10^{\wedge} 3$ ) for chlorahololide A (1) (labeled as ch2).

\begin{tabular}{|c|c|c|c|c|}
\hline & $\mathrm{x}$ & $\mathrm{y}$ & z & $\mathrm{U}(\mathrm{eq})$ \\
\hline $\mathrm{H}(1)$ & 8454 & 9900 & 9429 & 74 \\
\hline $\mathrm{H}(1 \mathrm{~A})$ & 6197 & 11933 & 7959 & 61 \\
\hline $\mathrm{H}(2 \mathrm{~A})$ & 6320 & 10568 & 9150 & 69 \\
\hline $\mathrm{H}(2 \mathrm{~B})$ & 7291 & 10908 & 9565 & 69 \\
\hline $\mathrm{H}(3)$ & 6569 & 9809 & 7842 & 64 \\
\hline $\mathrm{H}(9 \mathrm{~A})$ & 7112 & 12789 & 7052 & 67 \\
\hline $\mathrm{H}(9 \mathrm{~B})$ & 6903 & 13875 & 7567 & 67 \\
\hline $\mathrm{H}(13 \mathrm{~A})$ & 10724 & 13504 & 8828 & 70 \\
\hline $\mathrm{H}(13 \mathrm{~B})$ & 10232 & 12773 & 9325 & 70 \\
\hline $\mathrm{H}(13 \mathrm{C})$ & 10487 & 14112 & 9589 & 70 \\
\hline $\mathrm{H}(14 \mathrm{~A})$ & 7840 & 12757 & 9711 & 82 \\
\hline $\mathrm{H}(14 \mathrm{~B})$ & 7207 & 13729 & 9197 & 82 \\
\hline $\mathrm{H}(14 \mathrm{C})$ & 8122 & 13797 & 9214 & 82 \\
\hline $\mathrm{H}(15 \mathrm{~A})$ & 7644 & 10245 & 6961 & 61 \\
\hline $\mathrm{H}(15 \mathrm{~B})$ & 7800 & 8958 & 7385 & 61 \\
\hline $\mathrm{H}(16 \mathrm{~A})$ & 8665 & 17305 & 8364 & 163 \\
\hline $\mathrm{H}(16 \mathrm{~B})$ & 9503 & 17436 & 9115 & 163 \\
\hline $\mathrm{H}(16 \mathrm{C})$ & 8691 & 17256 & 9342 & 163 \\
\hline $\mathrm{H}(21)$ & 7874 & 8534 & 6121 & 89 \\
\hline $\mathrm{H}(22 \mathrm{~A})$ & 8962 & 8465 & 5061 & 132 \\
\hline $\mathrm{H}(22 \mathrm{~B})$ & 8094 & 7731 & 4828 & 132 \\
\hline $\mathrm{H}(23)$ & 7375 & 9613 & 4743 & 105 \\
\hline $\mathrm{H}(25)$ & 8392 & 11316 & 6293 & 69 \\
\hline $\mathrm{H}(26)$ & 9973 & 11606 & 6191 & 62 \\
\hline $\mathrm{H}(29)$ & 9155 & 9155 & 7815 & 56 \\
\hline $\mathrm{H}(33 \mathrm{~A})$ & 11347 & 12194 & 7224 & 99 \\
\hline $\mathrm{H}(33 \mathrm{~B})$ & 11781 & 11078 & 7775 & 99 \\
\hline $\mathrm{H}(33 \mathrm{C})$ & 11778 & 12324 & 8233 & 99 \\
\hline $\mathrm{H}(34 \mathrm{~A})$ & 9963 & 9311 & 6132 & 113 \\
\hline $\mathrm{H}(34 \mathrm{~B})$ & 9750 & 8231 & 6648 & 113 \\
\hline $\mathrm{H}(34 \mathrm{C})$ & 10202 & 9370 & 7151 & 113 \\
\hline $\mathrm{H}(35 \mathrm{~A})$ & 8235 & 11255 & 3941 & 131 \\
\hline $\mathrm{H}(35 \mathrm{~B})$ & 8836 & 12133 & 4659 & 131 \\
\hline $\mathrm{H}(37 \mathrm{~A})$ & 9060 & 15023 & 5352 & 137 \\
\hline $\mathrm{H}(37 \mathrm{~B})$ & 9828 & 15637 & 6017 & 137 \\
\hline $\mathrm{H}(37 \mathrm{C})$ & 9092 & 15223 & 6322 & 137 \\
\hline
\end{tabular}


Table 7. Torsion angles [deg] for chlorahololide A (1) (labeled as ch2).

\begin{tabular}{|c|c|}
\hline $\mathrm{C}(10)-\mathrm{C}(1)-\mathrm{C}(2)-\mathrm{C}(3)$ & $-99.6(4)$ \\
\hline $\mathrm{C}(1)-\mathrm{C}(2)-\mathrm{C}(3)-\mathrm{C}(4)$ & $97.5(4)$ \\
\hline $\mathrm{C}(10)-\mathrm{C}(1)-\mathrm{C}(3)-\mathrm{C}(2)$ & $110.0(4)$ \\
\hline $\mathrm{C}(2)-\mathrm{C}(1)-\mathrm{C}(3)-\mathrm{C}(4)$ & $-108.6(4)$ \\
\hline $\mathrm{C}(10)-\mathrm{C}(1)-\mathrm{C}(3)-\mathrm{C}(4)$ & $1.4(5)$ \\
\hline $\mathrm{C}(2)-\mathrm{C}(3)-\mathrm{C}(4)-\mathrm{O}(1)$ & $59.2(5)$ \\
\hline $\mathrm{C}(1)-\mathrm{C}(3)-\mathrm{C}(4)-\mathrm{O}(1)$ & $123.1(4)$ \\
\hline $\mathrm{C}(2)-\mathrm{C}(3)-\mathrm{C}(4)-\mathrm{C}(5)$ & $-66.9(4)$ \\
\hline $\mathrm{C}(1)-\mathrm{C}(3)-\mathrm{C}(4)-\mathrm{C}(5)$ & $-3.0(4)$ \\
\hline $\mathrm{C}(2)-\mathrm{C}(3)-\mathrm{C}(4)-\mathrm{C}(15)$ & $178.6(4)$ \\
\hline $\mathrm{C}(1)-\mathrm{C}(3)-\mathrm{C}(4)-\mathrm{C}(15)$ & $-117.5(4)$ \\
\hline $\mathrm{O}(1)-\mathrm{C}(4)-\mathrm{C}(5)-\mathrm{C}(6)$ & $75.5(5)$ \\
\hline $\mathrm{C}(15)-\mathrm{C}(4)-\mathrm{C}(5)-\mathrm{C}(6)$ & $-40.6(5)$ \\
\hline $\mathrm{C}(3)-\mathrm{C}(4)-\mathrm{C}(5)-\mathrm{C}(6)$ & $-159.6(3)$ \\
\hline $\mathrm{O}(1)-\mathrm{C}(4)-\mathrm{C}(5)-\mathrm{C}(10)$ & $-121.0(3)$ \\
\hline$C(15)-C(4)-C(5)-C(10)$ & $122.9(3)$ \\
\hline C(3)-C(4)-C(5)-C(10) & $3.8(4)$ \\
\hline $\mathrm{C}(4)-\mathrm{C}(5)-\mathrm{C}(6)-\mathrm{C}(7)$ & $169.4(3)$ \\
\hline $\mathrm{C}(10)-\mathrm{C}(5)-\mathrm{C}(6)-\mathrm{C}(7)$ & $7.3(5)$ \\
\hline C(4)-C(5)-C(6)-C(28) & $-6.4(5)$ \\
\hline $\mathrm{C}(10)-\mathrm{C}(5)-\mathrm{C}(6)-\mathrm{C}(28)$ & $-168.5(3)$ \\
\hline $\mathrm{C}(5)-\mathrm{C}(6)-\mathrm{C}(7)-\mathrm{C}(11)$ & $125.2(4)$ \\
\hline$C(28)-C(6)-C(7)-C(11)$ & $-59.3(5)$ \\
\hline $\mathrm{C}(5)-\mathrm{C}(6)-\mathrm{C}(7)-\mathrm{C}(8)$ & $-44.1(5)$ \\
\hline $\mathrm{C}(28)-\mathrm{C}(6)-\mathrm{C}(7)-\mathrm{C}(8)$ & $131.4(3)$ \\
\hline $\mathrm{C}(11)-\mathrm{C}(7)-\mathrm{C}(8)-\mathrm{O}(2)$ & $32.3(6)$ \\
\hline $\mathrm{C}(6)-\mathrm{C}(7)-\mathrm{C}(8)-\mathrm{O}(2)$ & $-157.2(4)$ \\
\hline $\mathrm{C}(11)-\mathrm{C}(7)-\mathrm{C}(8)-\mathrm{C}(9)$ & $-144.6(4)$ \\
\hline $\mathrm{C}(6)-\mathrm{C}(7)-\mathrm{C}(8)-\mathrm{C}(9)$ & $25.9(5)$ \\
\hline $\mathrm{O}(2)-\mathrm{C}(8)-\mathrm{C}(9)-\mathrm{C}(10)$ & $-151.2(4)$ \\
\hline $\mathrm{C}(7)-\mathrm{C}(8)-\mathrm{C}(9)-\mathrm{C}(10)$ & $25.7(5)$ \\
\hline $\mathrm{C}(2)-\mathrm{C}(1)-\mathrm{C}(10)-\mathrm{C}(5)$ & $67.1(4)$ \\
\hline $\mathrm{C}(3)-\mathrm{C}(1)-\mathrm{C}(10)-\mathrm{C}(5)$ & $0.9(4)$ \\
\hline $\mathrm{C}(2)-\mathrm{C}(1)-\mathrm{C}(10)-\mathrm{C}(9)$ & 179.1(4) \\
\hline C(3)-C(1)-C(10)-C(9) & $113.0(4)$ \\
\hline $\mathrm{C}(2)-\mathrm{C}(1)-\mathrm{C}(10)-\mathrm{C}(14)$ & $-57.8(5)$ \\
\hline $\mathrm{C}(3)-\mathrm{C}(1)-\mathrm{C}(10)-\mathrm{C}(14)$ & $-124.0(4)$ \\
\hline C(6)-C(5)-C(10)-C(1) & $160.6(3)$ \\
\hline $\mathrm{C}(4)-\mathrm{C}(5)-\mathrm{C}(10)-\mathrm{C}(1)$ & $-3.0(4)$ \\
\hline$C(6)-C(5)-C(10)-C(9)$ & $43.6(4)$ \\
\hline C(4)-C(5)-C(10)-C(9) & $-120.0(4)$ \\
\hline $\mathrm{C}(6)-\mathrm{C}(5)-\mathrm{C}(10)-\mathrm{C}(14)$ & $-77.3(5)$ \\
\hline $\mathrm{C}(4)-\mathrm{C}(5)-\mathrm{C}(10)-\mathrm{C}(14)$ & $119.1(4)$ \\
\hline $\mathrm{C}(8)-\mathrm{C}(9)-\mathrm{C}(10)-\mathrm{C}(1)$ & $-169.0(4)$ \\
\hline C(8)-C(9)-C(10)-C(5) & $-58.1(4)$ \\
\hline $\mathrm{C}(8)-\mathrm{C}(9)-\mathrm{C}(10)-\mathrm{C}(14)$ & $66.8(4)$ \\
\hline $\mathrm{C}(6)-\mathrm{C}(7)-\mathrm{C}(11)-\mathrm{C}(12)$ & $-172.5(4)$ \\
\hline $\mathrm{C}(8)-\mathrm{C}(7)-\mathrm{C}(11)-\mathrm{C}(12)$ & $-3.6(6)$ \\
\hline $\mathrm{C}(6)-\mathrm{C}(7)-\mathrm{C}(11)-\mathrm{C}(13)$ & $0.2(6)$ \\
\hline $\mathrm{C}(8)-\mathrm{C}(7)-\mathrm{C}(11)-\mathrm{C}(13)$ & $169.1(3)$ \\
\hline $\mathrm{C}(16)-\mathrm{O}(3)-\mathrm{C}(12)-\mathrm{O}(4)$ & 7.2(7) \\
\hline $\mathrm{C}(16)-\mathrm{O}(3)-\mathrm{C}(12)-\mathrm{C}(11)$ & $-178.4(5)$ \\
\hline $\mathrm{C}(7)-\mathrm{C}(11)-\mathrm{C}(12)-\mathrm{O}(4)$ & $-106.7(5)$ \\
\hline $\mathrm{C}(13)-\mathrm{C}(11)-\mathrm{C}(12)-\mathrm{O}(4)$ & $79.7(5)$ \\
\hline $\mathrm{C}(7)-\mathrm{C}(11)-\mathrm{C}(12)-\mathrm{O}(3)$ & $79.0(5)$ \\
\hline $\mathrm{C}(13)-\mathrm{C}(11)-\mathrm{C}(12)-\mathrm{O}(3)$ & $-94.6(4)$ \\
\hline $\mathrm{O}(1)-\mathrm{C}(4)-\mathrm{C}(15)-\mathrm{C}(29)$ & $-61.7(4)$ \\
\hline C(5)-C(4)-C(15)-C(29) & $61.2(4)$ \\
\hline
\end{tabular}




\begin{tabular}{|c|c|}
\hline C(3)-C(4)-C(15)-C(29) & 174.3(3) \\
\hline $\mathrm{C}(30)-\mathrm{C}(21)-\mathrm{C}(22)-\mathrm{C}(23)$ & $-96.2(6)$ \\
\hline $\mathrm{C}(21)-\mathrm{C}(22)-\mathrm{C}(23)-\mathrm{C}(24)$ & $95.4(5)$ \\
\hline $\mathrm{C}(30)-\mathrm{C}(21)-\mathrm{C}(23)-\mathrm{C}(22)$ & $113.0(5)$ \\
\hline $\mathrm{C}(22)-\mathrm{C}(21)-\mathrm{C}(23)-\mathrm{C}(24)$ & $-112.4(5)$ \\
\hline $\mathrm{C}(30)-\mathrm{C}(21)-\mathrm{C}(23)-\mathrm{C}(24)$ & $0.7(6)$ \\
\hline $\mathrm{C}(22)-\mathrm{C}(23)-\mathrm{C}(24)-\mathrm{C}(35)$ & $98.7(8)$ \\
\hline $\mathrm{C}(21)-\mathrm{C}(23)-\mathrm{C}(24)-\mathrm{C}(35)$ & $163.9(6)$ \\
\hline $\mathrm{C}(22)-\mathrm{C}(23)-\mathrm{C}(24)-\mathrm{C}(25)$ & $-84.0(5)$ \\
\hline $\mathrm{C}(21)-\mathrm{C}(23)-\mathrm{C}(24)-\mathrm{C}(25)$ & $-18.8(6)$ \\
\hline $\mathrm{C}(35)-\mathrm{C}(24)-\mathrm{C}(25)-\mathrm{C}(26)$ & $-21.7(9)$ \\
\hline $\mathrm{C}(23)-\mathrm{C}(24)-\mathrm{C}(25)-\mathrm{C}(26)$ & $161.0(5)$ \\
\hline $\mathrm{C}(35)-\mathrm{C}(24)-\mathrm{C}(25)-\mathrm{C}(30)$ & $-152.8(6)$ \\
\hline $\mathrm{C}(23)-\mathrm{C}(24)-\mathrm{C}(25)-\mathrm{C}(30)$ & $29.9(5)$ \\
\hline $\mathrm{C}(36)-\mathrm{O}(5)-\mathrm{C}(26)-\mathrm{C}(27)$ & $91.3(4)$ \\
\hline $\mathrm{C}(36)-\mathrm{O}(5)-\mathrm{C}(26)-\mathrm{C}(25)$ & $-151.3(4)$ \\
\hline $\mathrm{C}(24)-\mathrm{C}(25)-\mathrm{C}(26)-\mathrm{O}(5)$ & $73.4(5)$ \\
\hline $\mathrm{C}(30)-\mathrm{C}(25)-\mathrm{C}(26)-\mathrm{O}(5)$ & $-158.5(3)$ \\
\hline $\mathrm{C}(24)-\mathrm{C}(25)-\mathrm{C}(26)-\mathrm{C}(27)$ & $-165.8(4)$ \\
\hline $\mathrm{C}(30)-\mathrm{C}(25)-\mathrm{C}(26)-\mathrm{C}(27)$ & $-37.8(5)$ \\
\hline $\mathrm{O}(5)-\mathrm{C}(26)-\mathrm{C}(27)-\mathrm{C}(31)$ & $-103.2(5)$ \\
\hline $\mathrm{C}(25)-\mathrm{C}(26)-\mathrm{C}(27)-\mathrm{C}(31)$ & 139.1(4) \\
\hline $\mathrm{O}(5)-\mathrm{C}(26)-\mathrm{C}(27)-\mathrm{C}(28)$ & $92.7(4)$ \\
\hline $\mathrm{C}(25)-\mathrm{C}(26)-\mathrm{C}(27)-\mathrm{C}(28)$ & $-25.0(5)$ \\
\hline $\mathrm{C}(32)-\mathrm{O}(8)-\mathrm{C}(28)-\mathrm{C}(6)$ & $-119.0(3)$ \\
\hline $\mathrm{C}(32)-\mathrm{O}(8)-\mathrm{C}(28)-\mathrm{C}(27)$ & $0.3(4)$ \\
\hline $\mathrm{C}(32)-\mathrm{O}(8)-\mathrm{C}(28)-\mathrm{C}(29)$ & $119.0(3)$ \\
\hline $\mathrm{C}(5)-\mathrm{C}(6)-\mathrm{C}(28)-\mathrm{O}(8)$ & $-85.3(4)$ \\
\hline $\mathrm{C}(7)-\mathrm{C}(6)-\mathrm{C}(28)-\mathrm{O}(8)$ & $99.3(4)$ \\
\hline C(5)-C(6)-C(28)-C(27) & 162.1(3) \\
\hline$C(7)-C(6)-C(28)-C(27)$ & $-13.2(5)$ \\
\hline $\mathrm{C}(5)-\mathrm{C}(6)-\mathrm{C}(28)-\mathrm{C}(29)$ & $32.7(4)$ \\
\hline $\mathrm{C}(7)-\mathrm{C}(6)-\mathrm{C}(28)-\mathrm{C}(29)$ & $-142.7(3)$ \\
\hline $\mathrm{C}(31)-\mathrm{C}(27)-\mathrm{C}(28)-\mathrm{O}(8)$ & $-0.4(4)$ \\
\hline $\mathrm{C}(26)-\mathrm{C}(27)-\mathrm{C}(28)-\mathrm{O}(8)$ & $166.5(3)$ \\
\hline $\mathrm{C}(31)-\mathrm{C}(27)-\mathrm{C}(28)-\mathrm{C}(6)$ & $114.7(4)$ \\
\hline $\mathrm{C}(26)-\mathrm{C}(27)-\mathrm{C}(28)-\mathrm{C}(6)$ & $-78.4(4)$ \\
\hline $\mathrm{C}(31)-\mathrm{C}(27)-\mathrm{C}(28)-\mathrm{C}(29)$ & $-115.3(3)$ \\
\hline $\mathrm{C}(26)-\mathrm{C}(27)-\mathrm{C}(28)-\mathrm{C}(29)$ & $51.6(5)$ \\
\hline C(4)-C(15)-C(29)-C(30) & $-163.5(3)$ \\
\hline $\mathrm{C}(4)-\mathrm{C}(15)-\mathrm{C}(29)-\mathrm{C}(28)$ & $-37.0(5)$ \\
\hline $\mathrm{O}(8)-\mathrm{C}(28)-\mathrm{C}(29)-\mathrm{C}(15)$ & $108.2(3)$ \\
\hline C(6)-C(28)-C(29)-C(15) & $-9.7(4)$ \\
\hline $\mathrm{C}(27)-\mathrm{C}(28)-\mathrm{C}(29)-\mathrm{C}(15)$ & $-139.5(3)$ \\
\hline $\mathrm{O}(8)-\mathrm{C}(28)-\mathrm{C}(29)-\mathrm{C}(30)$ & $-123.9(3)$ \\
\hline C(6)-C(28)-C(29)-C(30) & $118.2(3)$ \\
\hline $\mathrm{C}(27)-\mathrm{C}(28)-\mathrm{C}(29)-\mathrm{C}(30)$ & $-11.6(4)$ \\
\hline $\mathrm{C}(23)-\mathrm{C}(21)-\mathrm{C}(30)-\mathrm{C}(25)$ & $17.1(5)$ \\
\hline $\mathrm{C}(22)-\mathrm{C}(21)-\mathrm{C}(30)-\mathrm{C}(25)$ & $82.1(6)$ \\
\hline $\mathrm{C}(23)-\mathrm{C}(21)-\mathrm{C}(30)-\mathrm{C}(34)$ & $-104.7(5)$ \\
\hline $\mathrm{C}(22)-\mathrm{C}(21)-\mathrm{C}(30)-\mathrm{C}(34)$ & $-39.8(7)$ \\
\hline $\mathrm{C}(23)-\mathrm{C}(21)-\mathrm{C}(30)-\mathrm{C}(29)$ & $132.1(4)$ \\
\hline $\mathrm{C}(22)-\mathrm{C}(21)-\mathrm{C}(30)-\mathrm{C}(29)$ & $-163.0(5)$ \\
\hline $\mathrm{C}(26)-\mathrm{C}(25)-\mathrm{C}(30)-\mathrm{C}(21)$ & $-164.0(3)$ \\
\hline$C(24)-C(25)-C(30)-C(21)$ & $-28.6(4)$ \\
\hline $\mathrm{C}(26)-\mathrm{C}(25)-\mathrm{C}(30)-\mathrm{C}(34)$ & $-46.3(5)$ \\
\hline $\mathrm{C}(24)-\mathrm{C}(25)-\mathrm{C}(30)-\mathrm{C}(34)$ & $89.2(4)$ \\
\hline $\mathrm{C}(26)-\mathrm{C}(25)-\mathrm{C}(30)-\mathrm{C}(29)$ & $75.0(4)$ \\
\hline $\mathrm{C}(24)-\mathrm{C}(25)-\mathrm{C}(30)-\mathrm{C}(29)$ & $-149.5(3)$ \\
\hline$C(15)-C(29)-C(30)-C(21)$ & $-27.8(5)$ \\
\hline $\mathrm{C}(28)-\mathrm{C}(29)-\mathrm{C}(30)-\mathrm{C}(21)$ & $-156.1(4)$ \\
\hline $\mathrm{C}(15)-\mathrm{C}(29)-\mathrm{C}(30)-\mathrm{C}(25)$ & $84.8(4)$ \\
\hline $\mathrm{C}(28)-\mathrm{C}(29)-\mathrm{C}(30)-\mathrm{C}(25)$ & $-43.5(4)$ \\
\hline
\end{tabular}


$\mathrm{C}(15)-\mathrm{C}(29)-\mathrm{C}(30)-\mathrm{C}(34)$

$\mathrm{C}(28)-\mathrm{C}(29)-\mathrm{C}(30)-\mathrm{C}(34)$

$\mathrm{C}(26)-\mathrm{C}(27)-\mathrm{C}(31)-\mathrm{C}(32)$

$\mathrm{C}(28)-\mathrm{C}(27)-\mathrm{C}(31)-\mathrm{C}(32)$

$\mathrm{C}(26)-\mathrm{C}(27)-\mathrm{C}(31)-\mathrm{C}(33)$

$\mathrm{C}(28)-\mathrm{C}(27)-\mathrm{C}(31)-\mathrm{C}(33)$

$\mathrm{C}(28)-\mathrm{O}(8)-\mathrm{C}(32)-\mathrm{O}(7)$

$\mathrm{C}(28)-\mathrm{O}(8)-\mathrm{C}(32)-\mathrm{C}(31)$

$\mathrm{C}(27)-\mathrm{C}(31)-\mathrm{C}(32)-\mathrm{O}(7)$

$\mathrm{C}(33)-\mathrm{C}(31)-\mathrm{C}(32)-\mathrm{O}(7)$

$\mathrm{C}(27)-\mathrm{C}(31)-\mathrm{C}(32)-\mathrm{O}(8)$

$\mathrm{C}(33)-\mathrm{C}(31)-\mathrm{C}(32)-\mathrm{O}(8)$

$\mathrm{C}(26)-\mathrm{O}(5)-\mathrm{C}(36)-\mathrm{O}(6)$

$\mathrm{C}(26)-\mathrm{O}(5)-\mathrm{C}(36)-\mathrm{C}(37)$
$-150.6(4)$

$81.1(4)$

$-165.1(4)$

$0.3(4)$

$12.9(8)$

178.3(4)

$-179.4(4)$

$-0.2(4)$

179.1(4)

$0.8(7)$

$-0.1(4)$

$-178.3(4)$

$-3.9(7)$

176.7(4)

Symmetry transformations used to generate equivalent atoms: 
Table 8. Hydrogen-bonds for chlorahololide A (1) (labeled as ch2) [A and deg.].

\begin{tabular}{|c|c|c|c|c|}
\hline D-H...A & $d(D-H)$ & $\mathrm{d}(\mathrm{H} \ldots \mathrm{A})$ & $d(D \ldots A)$ & $<(\mathrm{DHA})$ \\
\hline $\mathrm{C}(13)-\mathrm{H}(13 \mathrm{~B}) \ldots \mathrm{O}(7)$ & 0.96 & 2.96 & $3.587(5)$ & 124.3 \\
\hline $\mathrm{C}(13)-\mathrm{H}(13 \mathrm{~B}) \ldots \mathrm{O}(8)$ & 0.96 & 2.53 & $3.309(5)$ & 138.3 \\
\hline $\mathrm{C}(14)-\mathrm{H}(14 \mathrm{C}) \ldots \mathrm{O}(3)$ & 0.96 & 2.85 & $3.784(6)$ & 164.7 \\
\hline $\mathrm{C}(33)-\mathrm{H}(33 \mathrm{~A}) \ldots \mathrm{O}(6)$ & 0.96 & 2.36 & $3.197(7)$ & 145.0 \\
\hline $\mathrm{C}(35)-\mathrm{H}(35 \mathrm{~B}) \ldots \mathrm{O}(5)$ & 0.93 & 2.68 & $3.236(7)$ & 119.3 \\
\hline $\mathrm{C}(37)-\mathrm{H}(37 \mathrm{C}) \ldots \mathrm{O}(2)$ & 0.96 & 2.63 & $3.539(6)$ & 158.4 \\
\hline $\mathrm{C}(37)-\mathrm{H}(37 \mathrm{C}) \ldots \mathrm{O}(4)$ & 0.96 & 2.72 & $3.327(7)$ & 122.0 \\
\hline $\mathrm{C}(13)-\mathrm{H}(13 \mathrm{C}) \ldots \mathrm{O}(3) \# 1$ & 0.96 & 2.89 & $3.848(5)$ & 176.1 \\
\hline $\mathrm{C}(14)-\mathrm{H}(14 \mathrm{~A}) \ldots \mathrm{O}(7) \# 1$ & 0.96 & 2.90 & $3.757(6)$ & 150.0 \\
\hline $\mathrm{C}(2)-\mathrm{H}(2 \mathrm{~B}) \ldots \mathrm{O}(7) \# 1$ & 0.97 & 2.50 & $3.433(5)$ & 160.6 \\
\hline $\mathrm{O}(1)-\mathrm{H}(1) \ldots \mathrm{O}(7) \# 1$ & 0.82 & 2.03 & $2.844(4)$ & 175.6 \\
\hline $\mathrm{C}(1)-\mathrm{H}(1 \mathrm{~A}) \ldots \mathrm{O}(4) \# 2$ & 0.98 & 2.67 & $3.325(5)$ & 124.5 \\
\hline $\mathrm{C}(2)-\mathrm{H}(2 \mathrm{~A}) \ldots \mathrm{O}(4) \# 2$ & 0.97 & 2.71 & $3.348(5)$ & 123.7 \\
\hline $\mathrm{C}(3)-\mathrm{H}(3) \ldots \mathrm{O}(6) \# 2$ & 0.98 & 2.92 & $3.868(6)$ & 162.8 \\
\hline $\mathrm{C}(14)-\mathrm{H}(14 \mathrm{~B}) \ldots \mathrm{O}(7) \# 3$ & 0.96 & 2.72 & $3.637(5)$ & 159.3 \\
\hline $\mathrm{C}(33)-\mathrm{H}(33 \mathrm{~B}) \ldots \mathrm{O}(2) \# 4$ & 0.96 & 2.97 & $3.819(6)$ & 147.9 \\
\hline $\mathrm{C}(35)-\mathrm{H}(35 \mathrm{~A}) \ldots \mathrm{O}(2) \# 5$ & 0.93 & 2.86 & $3.667(7)$ & 145.2 \\
\hline $\mathrm{C}(35)-\mathrm{H}(35 \mathrm{~B}) \ldots \mathrm{O}(6) \# 6$ & 0.93 & 2.74 & $3.446(8)$ & 133.7 \\
\hline
\end{tabular}

Symmetry transformations used to generate equivalent atoms:

$\# 1-\mathrm{x}+2, \mathrm{y}, \mathrm{z}+2 \quad \# 2 \mathrm{x}-1 / 2, \mathrm{y}-1 / 2, \mathrm{z} \quad \# 3 \mathrm{x}-1 / 2, \mathrm{y}+1 / 2, \mathrm{z}$

$\# 4 \mathrm{x}+1 / 2, \mathrm{y}-1 / 2, \mathrm{z} \quad \# 5-\mathrm{x}+3 / 2, \mathrm{y}-1 / 2,-\mathrm{z}+1 \quad \# 6-\mathrm{x}+2, \mathrm{y},-\mathrm{z}+1$ 
Table 9. Least-squares planes (x,y,z in crystal coordinates) and deviations from them (* indicates atom used to define plane)

$15.3570(0.0299) \mathrm{x}+2.5514(0.0379) \mathrm{y}+1.3518(0.0569) \mathrm{z}=14.4561(0.0468)$

$\begin{array}{lll}* & 0.0000(0.0001) & \mathrm{C} 1 \\ * & 0.0000(0.0001) & \mathrm{C} 2 \\ * & 0.0000(0.0000) & \mathrm{C} 3 \\ & 1.3759(0.0068) & \mathrm{C} 4 \\ & 1.3303(0.0067) & \mathrm{C} 10\end{array}$

Rms deviation of fitted atoms $=\quad 0.0000$

$3.4373(0.0187) \mathrm{x}-0.0566(0.0150) \mathrm{y}+13.9852(0.0135) \mathrm{z}=14.2706(0.0215)$

Angle to previous plane (with approximate esd) $=56.08(0.26)$

* $\quad-0.0393(0.0023) \quad \mathrm{C} 4$

* $\quad 0.1136(0.0032) \quad \mathrm{C} 5$

* $\quad-0.0539(0.0022) \quad \mathrm{C} 10$

* $\quad 0.0484(0.0031) \quad \mathrm{C} 6$

* $\quad-0.0272(0.0022) \quad \mathrm{C} 7$

* $\quad-0.0417(0.0021) \quad \mathrm{C} 28$

Rms deviation of fitted atoms $=0.0608$

$-10.4907(0.0196) x+0.8376(0.0164) y+15.2445(0.0135) z=4.1511(0.0276)$

Angle to previous plane (with approximate esd) $=49.36(0.08)$

* $\quad 0.0158(0.0023) \quad$ C6

* $\quad-0.0752(0.0032) \quad \mathrm{C} 7$

* $\quad 0.0573(0.0024) \quad \mathrm{C} 8$

* $\quad-0.0584(0.0034) \quad \mathrm{C} 11$

* $\quad 0.0544(0.0023) \quad \mathrm{C} 13$

* $\quad 0.0061(0.0026) \quad \mathrm{C} 12$

Rms deviation of fitted atoms $=\quad 0.0510$

$-4.4157(0.0396) \mathrm{x}+5.2838(0.0227) \mathrm{y}+14.2088(0.0207) \mathrm{z}=14.5415(0.0420)$

Angle to previous plane (with approximate esd) $=31.14(0.18)$

* $\quad-0.0165(0.0036) \quad \mathrm{C} 8$

* $\quad 0.0066(0.0014) \quad \mathrm{O} 2$

* $\quad 0.0049(0.0011) \quad \mathrm{C} 7$

* $\quad 0.0050(0.0011) \quad$ C9

Rms deviation of fitted atoms $=0.0096$

$10.9525(0.0257) x+0.6735(0.0523) y+8.5006(0.0267) z=18.5936(0.0790)$

Angle to previous plane (with approximate esd $)=59.18(0.21)$

* $\quad-0.0300(0.0027) \quad \mathrm{C} 12$

* $\quad-0.0121(0.0011) \quad \mathrm{C} 16$ 
* $\quad 0.0260(0.0024) \quad \mathrm{O} 3$

* $\quad 0.0161(0.0015) \quad \mathrm{O} 4$

Rms deviation of fitted atoms $=0.0223$

$14.4522(0.0557) \mathrm{x}+5.5101(0.0537) \mathrm{y}-0.9830(0.0822) \mathrm{z}=16.3011(0.0333)$

Angle to previous plane (with approximate esd) $=42.89(0.42)$

* $\quad 0.0000(0.0001) \quad \mathrm{C} 21$

* $\quad 0.0000(0.0001) \quad \mathrm{C} 22$

* $\quad 0.0000(0.0000) \quad \mathrm{C} 23$

$1.3272(0.0092) \quad \mathrm{C} 24$

$1.3406(0.0090) \quad \mathrm{C} 30$

Rms deviation of fitted atoms $=0.0000$

$14.7122(0.0281) x-5.7457(0.0284) y-4.8230(0.0413) z=3.7687(0.0460)$

Angle to previous plane (with approximate esd) $=63.03(0.27)$

* $\quad 0.0048(0.0022) \quad \mathrm{C} 23$

* $\quad 0.0177(0.0054) \quad \mathrm{C} 24$

* $\quad-0.0169(0.0019) \quad \mathrm{C} 25$

* $\quad 0.0019(0.0014) \quad \mathrm{C} 35$

* $\quad-0.0201(0.0017) \quad \mathrm{H} 35 \mathrm{~A}$

* $\quad 0.0126(0.0021) \quad \mathrm{H} 35 \mathrm{~B}$

Rms deviation of fitted atoms $=0.0141$

$-3.4402(0.0283) x+10.3168(0.0103) y+5.7004(0.0265) z=12.6369(0.0300)$

Angle to previous plane (with approximate esd) $=48.56(0.21)$

* $\quad-0.0852(0.0022) \quad \mathrm{C} 26$

* $\quad 0.0931(0.0034) \quad \mathrm{C} 27$

* $\quad 0.0276(0.0027) \quad \mathrm{C} 28$

* $\quad 0.0303(0.0037) \quad \mathrm{C} 31$

* $\quad-0.0843(0.0026) \quad \mathrm{C} 32$

* $0.0185(0.0024) \quad$ C33

Rms deviation of fitted atoms $=0.0646$

$-3.3285(0.0304) x+9.9881(0.0124) y+6.9287(0.0310) z=13.3858(0.0335)$

Angle to previous plane (with approximate esd) $=5.03(0.20)$

* $\quad-0.0012(0.0010) \quad \mathrm{C} 31$

* $\quad 0.0041(0.0033) \quad \mathrm{C} 32$

* $\quad-0.0017(0.0013) \quad 07$

* $\quad-0.0012(0.0010) \quad 08$

Rms deviation of fitted atoms $=0.0024$

$-1.2377(0.0487) x+2.6292(0.0339) y+15.2183(0.0176) z=11.8386(0.0478)$

Angle to previous plane (with approximate esd) $=52.94(0.24)$ 
* $\quad-0.0034(0.0045) \quad \mathrm{C} 36$

* $\quad 0.0010(0.0013) \quad \mathrm{C} 37$

* $\quad 0.0010(0.0014) \quad$ O5

* $\quad 0.0013(0.0018) \quad$ O6

Rms deviation of fitted atoms $=\quad 0.0020$ 